\title{
The Deep Impact Earth-Based Campaign
}

\author{
K. J. Meech* \\ Univ. Hawaii, Institute for Astronomy, 2680 Woodlawn Drive, Honolulu, HI 96822 \\ M. F. A'Hearn \\ Dept. of Astronomy, Univ. Maryland, College Park MD 20742-2421 \\ Y. R. Fernández \\ Univ. Hawaii, Institute for Astornomy, 2680 Woodlawn Drive, Honolulu, HI 96822
}

C. M. Lisse, H. A. Weaver

Johns Hopkins Univ.; APL, Space Dept., 11100 Johns Hopkins Road, Laurel, MD 20723-6099

N. Biver

Observatoire de Paris-Meudon, 5 Place Jules Jansses, 92190 Meudon, France

and

L. M. Woodney

Dept. of Physics. Univ. of Central Florida, P.O. Box 162385, Orlando, FL 32816-2385

* Author for correspondence: E-mail: meech@ifa.hawaii.edu

(Received September 15 2004; Accepted in final form February 3 2005)

\begin{abstract}
Prior to the selection of the comet 9P/Tempel 1 as the Deep Impact mission target, the comet was not well-observed. From 1999 through the present there has been an intensive world-wide observing campaign designed to obtain mission critical information about the target nucleus, including the nucleus size, albedo, rotation rate, rotation state, phase function, and the development of the dust and gas coma. The specific observing schemes used to obtain this information and the resources needed are presented here. The Deep Impact mission is unique in that part of the mission observations will rely on an Earth-based (ground and orbital) suite of complementary observations of the comet just prior to impact and in the weeks following. While the impact should result in new cometary activity, the actual physical outcome is uncertain, and the Earth-based observations must allow for a wide range of post-impact phenomena. A world-wide coordinated effort for these observations is described.
\end{abstract}

Keywords: Deep Impact, Earth observing 


\section{Introduction}

The selection of the Deep Impact mission target was driven primarily by launch and orbital dynamics considerations (A'Hearn et al. 2005; Blume 2005) and not by what was known about the potential target. Nevertheless, the success of the mission is greatly enhanced if much is known about the target a-priori. This is of benefit not only for planning the encounter sequences, but is especially important in the case of the Deep Impact mission target, $9 \mathrm{P} /$ Tempel 1, because the goal is to look for post-impact changes in the outgassing which will be indicative of the properties of the pristine nucleus interior. To this extent, more so than for any other mission, it is very important to establish a good baseline of observations which characterize the nucleus prior to the encounter. Furthermore the pre-impact ground observations will verify the capability of ground-based techniques for basic cometary nucleus reconnaissance.

The Deep Impact mission has been designed to provide good observing conditions from Earth. Although the flyby spacecraft of the Deep Impact mission will make unique in-situ measurements, the constraints of space missions limit us to imaging and near-infrared spectroscopy in an 800-sec interval from time of impact until the flyby spacecraft has flown past the point of observability of the impact site. A unique aspect of this mission is the observing program planned from Earth and Earth-orbit at the time of impact. These observations are designed to complement the spacecraft data during the period surrounding encounter, and will continue long after the event, since long-lived changes in the behavior are a plausible outcome of the experiment. However it is important to note that the specific outcome of the impact is unknown; indeed, that is one significant motivation for the mission in the first place. We and our collaborators have set up a ground-based observing campaign that will allow us to observe whatever phenomena are created by the impact.

\subsection{Selection of DI Target - What we Knew}

At the time of target selection, 9P/Tempel 1 was known to be a typical Jupiter-family lowactivity comet (A'Hearn et al. 1995) rarely bright as seen from Earth, and was well placed for ob- serving only every other apparition ( 11 year intervals). Although the comet was discovered in 1867 (Yeomans et al. 2005), there have thus been relatively few physical observations of the comet prior to its selection for the Deep Impact mission. From the inclusion of the non-gravitational parameters in the orbit solution for the comet, it was suggested that the pattern of outgassing was relatively unchanged during the last 7 apparitions. Most of the existing physical observations were of the gas and dust coma around the time of the two perihelion passages of 1983 and 1994 (Lisse et al. 2005), along with some limited information about the nucleus rotation (Belton et al. 2005).

In order to prepare for the encounter, the Deep Impact team has undertaken a large observing campaign of nucleus characterization. The sections below discuss the rationale and opportunities for the observations; many of the results are presented in Belton et al. (2005).

\section{The Pre-Encounter Period}

\subsection{Size and Albedo}

Knowledge of the size and shape of the nucleus and its albedo is important for the autonomous targeting software and in order to calculate instrument exposure times. To ensure targeting that is not too close to the limb such that the impactor might miss, it is crucial to know the size and shape of the nucleus. If the albedo is higher than assumed, the size will be correspondingly smaller and we would have to adopt a strategy that ensures that we hit the target without optimizing the observability. If the albedo is lower than assumed, the size will be correspondingly larger and we can optimize the targeting for observability of the crater at closest approach.

Simultaneous optical and thermal infrared observations of the bare nucleus can give an estimate of both the instantaneous nucleus size and geometric albedo. The technique relies on the fact that the flux in the optical is proportional to the nucleus cross section, albedo and phase function, whereas the thermal flux is related to the nucleus size, thermal phase function and nucleus emissivity (Lebofsky \& Spencer, 1989). As seen in Figure 1 , which is a light curve combining CCD data since 1999 with data from the International Comet Quarterly, the onset of activity typically occurs for 
this comet between 600-400 days pre-perihelion, at a heliocentric distance $r$ around 3 to 4 AU. However, signal-to-noise calculations for detecting a nucleus of the size of $9 \mathrm{P} /$ Tempel 1 in the thermal IR at the Keck 10m telescope indicated that this would only be feasible inside $r<2.5 \mathrm{AU}$, at which time the comet would be active.

Thus, when completely devoid of coma, the nucleus would be too faint to detect in the thermal IR even with the 10-m Keck telescope. Therefore a compromise was made, and data were obtained post-perihelion in August 2000 when the comet was at $r=2.54 \mathrm{AU}$ and still very active, necessitating significant modeling to remove the coma contribution and to determine the rotational phase at the time of observations (Fernández et al. 2003; Belton et al. 2005).

The only other opportunity to obtain data on the bare nucleus meant using the Spitzer Space Telescope. Director's discretionary time was awarded for this project so that we could obtain rotationally resolved IR fluxes during early 2004. Table 1 presents a summary of the opportunities for observing the comet that are compatible with the Spitzer Space Telescope observing windows. We were also awared General Observer time in Cycle 1 to observe the comet in 2005 after activity had started.

\subsection{Rotation State}

Observed brightness variations of a comet may be caused by: the activity, which changes the effective scattering area; the changing geocentric and heliocentric distances; the rotation of the nucleus (contributing a brightness modulation due to changing shape or surface albedos); and the changing solar illumination and scattering angles. Knowledge of the rotation period is important both for thermal modeling, as well as mission planning. In addition, the rotation state is a fundamental property of the nucleus and an understanding of the rotation can be used to derive constraints on the nucleus bulk strength and density. We need to be able to ensure that the impact occurs on the largest face of the nucleus (giving a higher probability for success), and the last time to totally control the time of impact was at launch. Now after launch, we are able to make only small adjustments because of fuel limitations.
Inverting a photometric light curve for an asteroid or an inactive comet is non-trivial. In increasing order of difficulty, one can obtain the $(i)$ sidereal rotation period and spin axis, (ii) the shape; and (iii) the light scattering properties (Magnusson et al. 1989). Ideally, the nucleus should be inactive, but as bright as possible and near opposition in order to obtain the maximum observing time per night. In order to place constraints on the rotation pole, we want at least four good light curves without coma contamination; a unique pole solution can be obtained in the ideal case only with at least three light curves. These should be at low phase angle $\left(\alpha<20^{\circ}\right.$, although it is better if $\alpha<10^{\circ}$ ) so that it is possible to associate light curve features with the shape of the nucleus rather than the specifics of the light scattering from the particulate surface. In addition, we should obtain the observations over a range of ecliptic longitudes in order to sample different geometries. Unfortunately, most comets begin to become active out near $r \sim 5-6$ AU (Meech \& Svoreň 2005), and as the nuclei are quite small with low albedos (Meech et al. 2004), this means that the inactive nucleus is quite faint, requiring large telescopes to achieve observations of adequate $\mathrm{S} / \mathrm{N}$.

The best periods for obtaining data for the rotational light curve are shown in Table 2. Because the rotation period was known to be long and that aliasing effects from the daily sampling could be a problem, we tried to coordinate observations between observatories separated by longitude. Intensive international observing campaigns were conducted during the 2000, 2001 and 2002 opportunities. The results of these efforts are discussed in (Belton et al. 2005).

\subsection{Phase Function}

Most bodies in the solar system with particulate light-reflecting surfaces have been observed to exhibit a nonlinear surge in brightness at low phase angles $\left(\alpha<7^{\circ}\right)$. This is caused by a combination of particles in the surface covering their shadows and a contribution from coherent backscattering. In principle, observations over a wide range of $\alpha$ can be used with detailed photometric models of the multiply scattered light in rough particulate surfaces to get information about the particle single scattering albedos, the porosity of the surface, the particle sizes, compaction of the sur- 
face and the macroscopic roughness of the surface. Detailed modeling requires high-quality data over a wide range of phase angles. The parameters which describe the small-scale surface scattering properties of the optically active regolith (porosity, particle size distribution, and compaction) can be well constrained from observations at phase angles $\alpha<12^{\circ}$, whereas macroscopic roughness can only be ascertained by large phase angle observations. Less compacted, fluffy regoliths have more pronounced surges. Macroscopically rough features (clumps to mountains, craters and ridges), can alter the local incidence and emergence angles which has an effect on brightness. This term can provide information about the impact and outgassing history of the nucleus and its effect on topology. For comets, knowledge of the particle sizes in the surface helps in the understanding of the degree to which the surface has been subjected to microphysical processes, micrometeorite impacts and annealing, and will be important for understanding and modeling the heat transfer into the nucleus.

For comets these observations are particularly difficult because not only is knowledge of the rotation important (to remove its signature), but the comet must not have any activity to compete with the small changes in brightness with phase. The phase-dependent photometric behavior of comets is currently poorly understood. There have been attempts to determine the phase functions for the nuclei of $2 \mathrm{P} /$ Encke and $28 \mathrm{P} /$ Neujmin 1 (Fernández et al. 2000; Delahodde et al. 2001) from the ground and 19P/Borrelly (Buratti et al. 2004) in-situ. Both for the practical issues related to understanding the likely brightness of the nucleus at the high phase angles at encounter as seen from the spacecraft $\left(63^{\circ}\right)$ and to understand the nucleus surface properties prior to impact, there were two observing seasons pre-encounter for which it was possible to undertake a campaign to acquire the necessary phase function data. Observations require that the comet is without coma, and that there is good coverage of the phase curve (at least 3-4 data points between about $15^{\circ}$ to $2-3^{\circ}$, and then dense coverage for $\alpha<2^{\circ}$ ). Furthermore, the comet should be at opposition so that long time-series observations can be made to remove the rotational signature from the phase function data.
The epochs and geometric circumstances are shown in Table 3. A major international campaign was undertaken for the Fall 2000 opportunity, producing an excellent set of data. However, because of weather, insufficient information was obtained to remove the rotation signature using this data set alone. Although also plagued again by poor weather, data from the Fall 2003 campaign have been calibrated and should yield good phase function data as the rotational state is solved. This phase function analysis is ongoing (Hsieh et al., personal communication).

\subsection{Gas Production}

\subsubsection{Optical, Ultraviolet and Infrared Wave- lengths}

The surfaces of comets are mostly inactive, and because of this, the impactor will probably hit an inactive region. The likely outcome of the impact is that the crater will become a new active area, which will lead to new outgassing, lasting days to months. During the campaign to observe the Shoemaker-Levy 9 impact into Jupiter, there were many pre-impact predictions. Many spectroscopic observations showed emissions from unexpected species, and there were a number of expected emission features that were absent (Noll et al. 1995). The goal of observing the gas production is to determine the changes in natural activity due to the impact. At optical wavelengths, this means monitoring the onset and development of

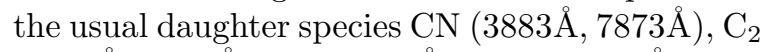
$(5165 \AA, 7715 \AA), \mathrm{C}_{3}(4040 \AA), \mathrm{NH}_{2}(5700 \AA)$, and $\mathrm{CH}(4315 \AA)$. These species have been monitorable from large telescopes since October / November 2004, when the comet left solar conjunction. The comet had a gas production rate of approximately $3 \times 10^{27}$ molecules per second (A'Hearn et al. 1995).

From spectroscopic surveys of comets in the optical, comet 9P/Tempel 1 has been found to have typical composition ratios of $\mathrm{OH}, \mathrm{CN}$ and $\mathrm{C}_{2}$ (A'Hearn et al. 1995). We therefore expect the ultraviolet spectrum of the comet to be similar to other comets that have been observed in the UV by both HST and IUE. These observations will be possible only near the time of encounter, when the comet is bright (see $\S 4.3 .10$ ). In the near-IR spectral region, spectral observations will only be 
possible for this comet at encounter.

\subsubsection{Radio and sub-mm wavelengths}

The molecules of primary interest in the radio and sub-mm wavelength regimes are $\mathrm{H}_{2} \mathrm{O}(\mathrm{OH})$, $\mathrm{CO}, \mathrm{CH}_{3} \mathrm{OH}$ and $\mathrm{HCN} . \mathrm{H}_{2} \mathrm{O}$ is the main driver of cometary activity, and one rotational line at $557 \mathrm{GHz}$ is currently observable from space satellites, while its main photo-dissociation product, $\mathrm{OH}$, is observable from the ground at $18 \mathrm{~cm}(1.7$ $\mathrm{GHz}$ ) or with narrowband UV filters on optical telescopes. The brightness of the $\mathrm{OH}$ lines is related to the total production of water in the coma and the spectral line shape contains information on the velocity of the outflowing gas, and the distribution of gas production from the nucleus. $\mathrm{CO}$ abundance relative to water is very variable from comet to comet (1-25\%) and can be depleted in the outer layers of cometary nuclei due to its high volatility. Methanol $\left(\mathrm{CH}_{3} \mathrm{OH}\right)$ is the 4 th most abundant molecule in comets (after $\mathrm{H}_{2} \mathrm{O}, \mathrm{CO}$ and $\mathrm{CO}_{2}$ ), sometimes even more abundant than $\mathrm{CO}$, and has been detected in the sub-mm in over 15 comets (Biver et al. 2002). Methanol lines are numerous and some can be observed simultaneously to probe the cometary gas temperature.

$\mathrm{HCN}$ is of lesser abundance relative to water ( $0.1 \%$ on average) but it is generally the easiest species detectable in comets from ground based sub-mm observatories. $\mathrm{HCN}$ can be observed via vibrational transitions in the infrared or via rotational transitions in the millimeter $(\mathrm{J}=1-0$ at $88.6 \mathrm{GHz} / 3.4 \mathrm{~mm})$ and submillimeter $(\mathrm{J}=3-2$ at $266 \mathrm{GHz} / 1.1 \mathrm{~mm}$ or $\mathrm{J}=4-3$ at $355 \mathrm{GHz} / 0.85 \mathrm{~mm}$ ). It has been detected in over 24 comets. Its abundance relative to water also displays a smaller variation than for $\mathrm{CO}$ and $\mathrm{CH}_{3} \mathrm{OH}$ and thus it constitutes a good reference. $9 \mathrm{P} /$ Tempel 1 is not intrinsically bright, so these observations can begin only as the comet approaches perihelion.

$\mathrm{OH}$ measurements will just be possible between March and May 2005. The $\mathrm{OH} 18-\mathrm{cm}$ maser line arises from the ground state $\Lambda$ doublet. The excitation of the two energy levels is dominated by pumping by solar UV radiation (a UV photon is absorbed followed by rapid cascading to the ground state) and depends on the relative velocity between the comet and the sun. This process leads to either strong inversion $\left(i=\left(n_{\text {up }}-n_{\text {low }}\right) /\right.$ $\left.\left(n_{\text {up }}+n_{\text {low }}\right)>0\right)$ or anti-inversion $(i<0)$ of the population of the $\Lambda$ doublet and the cometary $\mathrm{OH}$ coma then either amplifies or absorbs the background radiation. The pumping process strongly depends on the solar spectrum seen by the comet. Due to the complex structure of solar UV spectrum, the cometary velocity relative to the Sun will determine the inversion value. $\mathrm{OH}$ line intensities are proportional to $i$ in the first approximation, which will be between $-0.3<i<-0.2$ in March to May 2005 but near $i=0.02$ at perihelion. Under normal activity, $\mathrm{OH}$ lines in comet 9P/Tempel 1 will only be detectable before perihelion. A ten-fold increase in production rate from the impact (or an unusual excitation process) will be necessary to detect $\mathrm{OH}$. Daily observations of $\mathrm{OH}$ at Nançay (France) can be done, $1 \mathrm{~h}$ per day when the comet is close to meridian. Other facilities like the VLA (USA) and Parkes (Australia) should be able to observe the $\mathrm{OH}$ lines in comet 9P/Tempel 1 a few hours per day.

The HCN(3-2) or HCN(4-3) lines are expected to be detectable most of the time between April and July $2005(\mathrm{~S} / \mathrm{N}>5$ per observing run for any sub-mm telescope) for "normal" cometary activity. HCN observations will thus be useful to monitor cometary activity and enable precise measurement of the gas outflow velocity thanks to a good $\mathrm{S} / \mathrm{N}$ ratio and the very high spectral resolution of the heterodyne technique. Because of intrinsically weak lines, CO may not be detectable before impact if its abundance relative to water is below $5 \%$. Methanol should be detectable with long integration times to retrieve the gas temperature, while other molecular species like $\mathrm{CS}, \mathrm{H}_{2} \mathrm{~S}, \mathrm{H}_{2} \mathrm{CO}$, will likely be marginally detectable in the assumption of classical abundances and normal activity of comet 9P/Tempel 1.

A list of expected line strengths for some radio emission lines in commonly observed species is given in Table 4. Note that these calculations apply to the comet before impact. The baseline prediction is for the comet to become several times brighter immediately after impact in which case the line strengths will likewise increase. The signal-to-noise ratios $S / N$ given in the table assume that the line is observed for about 2.4 hours.

\subsection{Dust Development}

We want to closely monitor the dust environment around the nucleus ( $i$ ) to interpret other ob- 
servations with respect to the amount of coma contamination; ( $i$ ) to predict the brightness and dust production pre-impact for comparison post impact; ( iii) to model (using Finson-Probstein techniques) the near-nucleus dust environment and assess the impact hazard to the spacecraft.

Comet 46P/Wirtanen began its activity between $r=3.5-4.5$ AU pre-perihelion and 81P/Wild 2 began to develop a coma between $r=4.0$ 4.5 AU, based on dust production models for the Stardust mission. Assuming similar behavior for 9P/Tempel 1, observations were planned regularly from aphelion at $r=4.7 \mathrm{AU}(10 / 2002)$ to $3.5 \mathrm{AU}$ $(05 / 2004)$, every 1 to 2 months to get deep images to assess when activity first began.

\subsubsection{Finson-Probstein Dust modeling}

From past apparitions, this comet typically exhibits a sharp rise in brightness caused by outgassing and development of an extensive dust coma $\sim 200$ days pre-perihelion (although lower levels of activity begin much sooner; see Fig. 1). This occured in Dec. 2004 ( $r=2.5-2.3$ AU). Images taken on December 1 show a dust coma extending out beyond $2 \times 10^{4} \mathrm{~km}$ from the nucleus (Fig. 2). By observing the extent and morphology of the dust coma and using dust-dynamical models (Finson and Probstein 1968) we can determine the relative velocity distribution, size distribution and production rates of the dust as a function of distance. These models evaluate the motion of a suite of particles after leaving the nucleus under the influence of solar radiation pressure and gravity. The scattered light from the dust is added together and fit to the surface brightness of the observed coma. The larger the extent of the detected coma (i.e. observations must go to low surface brightness levels above sky, dictating dark time), the more constraining the models.

9P/Tempel 1 is a good comet to model because beginning in February 2005 through encounter there are large orbital geometry changes from month to month. Although the geometry is not as good in the fall 2004 (we are looking edge on to the tail and cannot separate the motions of the different grains), we needed to establish our first data point as a baseline for the onset of dust production as soon as the comet came out of solar conjunction in late October 2004. Some of the specific criteria for the optimization of wide field
R-band images of the dust coma include:

- Small particles move rapidly away from the field of view, so we need many images closely spaced in time to follow them.

- Large grains, which move slowly along the orbit, need observations equally spaced over time periods of months for proper modeling. The large dust is of particular interest because of the potential hazard to the spacecraft as it passes through the orbit plane approximately 15 minutes after impact.

- We would like to have the predicted dust trajectories (syn-curves) widely spaced in time to get better constraints on the particle sizes, dust ejection velocities, and dust production start and end.

- Small particles tend to lie in anti-solar direction whereas large particles lag behind in orbit.

- Very circular orbits tend to have widely spaced syn-curves, but it is then harder to see changes. Comets on very elliptical orbits have problems inbound when the large particles also fall along the sun-to-comet radius vector (and overlap the syn-curves for small ones). 9P/Tempel 1 is a good comet in that its orbit has an intermediate eccentricity.

It is anticipated that as the comet brightens in the spring of 2005, the Small Telescope Science Program observers (McFadden et al. 2005) will contribute greatly to the data set of wide field dust images needed for the modeling.

\subsubsection{Features in the Dust Coma}

The Earth passes through 9P/Tempel 1's orbit plane twice a year, on roughly May 31 and December 1 ( \pm 1 day). At these times we have the best chance for imaging the dust trail of large particles from previous apparitions, as has been done optically for comets $22 \mathrm{P} /$ Kopff and $81 \mathrm{P} /$ Wild 2 (Ishiguro et al. 2002, 2003). Finson-Probstein dust-dynamical analysis of the grains in both dust trails suggested particles between millimeter-tocentimeter sizes. A peak intensity at a mean 
anomaly $0.02^{\circ}$ from the comet for $22 \mathrm{P} /$ Kopff suggested that most of the large dust was emitted near perihelion.

The IRAS satellite detected a dust trail for 9P/Tempel 1 (Sykes \& Walker 1992), however, no optical trail has been found so far for $9 \mathrm{P} /$ Tempel 1. Knowledge of the dust distribution in the trail for $9 \mathrm{P} /$ Tempel 1, i.e. if most of the hazard is from material emitted at perihelion, will be important for the Deep Impact mission. We crossed the orbit plane of the comet on $12 / 1 / 04$, and we observed the comet at the UH $2.2 \mathrm{~m}$ telescope (Fig. 2) to do deep imaging to search for the trail. We will also use the Spitzer telescope (see Section 4.3.10 below) dust observations in 2005 to look for the dust trail.

Figure 3 summarizes the visibility of the comet since the time of the mission selection, through the year of impact. The periods of visibility are shown as a function of the changing heliocentric distance of the comet by year (as arrows). The shaded part of the figure roughly shows the time period when the comet is expected to possess significant coma. Letters on the figure indicate when there were or will be targeted campaigns to measure the comets rotation (R), dust coma development or disappearance (D), onset of gas production (S), and albedo/size measurements (A). It can be seen that the 2000 perihelion passage was not well suited for observations of the active comet, but that we had excellent access to the inactive phases of the comet near aphelion.

\section{Status of the Observations}

\subsection{Summary of the Observing Runs}

To date, we and our collaborators have obtained optical imaging data on 250 nights using 13 telescopes at 9 ground-based observatories world-wide in order to characterize the nucleus rotation state, size, albedo and dust development, in addition to data from the Keck $10 \mathrm{~m}$ and Spitzer telescope for albedo and nucleus size measurement and the Hubble Space Telescope for rotation determination. The level of cooperation among planetary colleagues as well as from colleagues outside the field has been excellent. In additional to our formal collaborators, there have been many observers who have given up some large telescope time in order to help out the mission, and they have made a tremendous contribution to our understanding of the nucleus properties. A summary of these runs through 2004 is included in Table 5.

\subsection{Development of the DI Database}

In order to facilitate access to the growing amount of Deep Impact ground-based imaging, we designed and developed a relational database using IBM's DB2 database management system, which can be queried using a web interface developed in ColdFusion MX.

In its current form, the database contains reduced data products (photometry) from the ground-based CCD imaging program. Routines developed in perl are used to parse the text files containing reduced data and insert the data into the database. Full information about the observations obtained on each night, including information about the observers and observing conditions, seeing, the instrument and telescope used (along with filter transmission curves and CCD quantum efficiencies) are stored in the database. Each observation is also tagged with the instantaneous values of the heliocentric and geocentric distances $(r$ and $\Delta)$ and phase angle $(\alpha)$, as well as the suncentered and Earth-centered state vectors. These values are obtained automatically by communicating with the Jet Propulsion Laboratory Solar System Dynamics ephemeris routines when new data is uploaded to the database.

This Deep Impact database can be queried using a password protected web interface (see Figure 4). The returned data can be sorted on several key parameters, such as date, time, filter, photometry aperture, by telescope, observer or instrument, and returned as either an HTML table, tabdelimited dataset, an excel spreadsheet or CSV file format.

\section{Coordination During the Encounter}

The goal of having a focal point of coordination for the mission, rather than allowing a completely open competitive process drive the Earth-based science, is to ensure that mission-critical observations are made which are necessary for the interpretation of the spacecraft data, and to avoid duplication of effort.

At the time of encounter, the impact will be observable in dark skies from longitudes as far west 
as New Zealand, and as far east as Arizona. Latitudes farther north than $\phi>50^{\circ}$ will not be able to observe the comet at all, as it will have a southern declination $\left(\mathrm{RA}=13^{h} 37^{m} 52^{s}, \delta=-09^{\circ} 4^{\prime} 07^{\prime \prime}\right)$, and because it will not get completely dark at these latitudes. The observing circumstances for major world-wide facilities, arranged in order of longitude are shown in Table 6 . New moon occurs on 6 July 2005, so the encounter will occur during dark skies (lunar illumination 4.9\%). The comet will be $129^{\circ}$ away from the moon, at a galactic latitude of $52^{\circ}$.

\subsection{The Observing Workshops}

In order to educate the astronomical community about both the science goals of the Deep Impact mission, as well as the technical challenges, the spacecraft observing constraints, and the desired ground-based observations, a series of community workshops were organized. The goals of these workshops were:

- To update the community on the status of the mission and the science goals;

- To discuss the key ground support observations that will be needed at various wavelengths at the time of encounter;

- To discuss the unique capabilities of worldwide facilities to participate in the Deep Impact science;

- To form collaborations and begin the work for writing key project proposals for both the pre-impact science (November 2004 through impact) and the encounter science. Most major observatory proposal deadlines for the encounter period were late September/early October 2004; and

- To make the different observatories aware of the public interest in the mission, and share some of the Education and Public Outreach materials that have been developed for the mission.

Table 7 summarizes the workshops that have been held.

\subsubsection{The DI Collaborator Webpage}

As a result of the workshops, a series of web pages for Deep Impact collaborators have been developed: http://deepscience.astro.umd.edu/collab/. The purpose of the web site is to support and coordinate the Earth-based observing program, by providing the observing community with information necessary for proposal writing; giving the community access to meeting presentations, as well as information about the instruments and facilities available at different observatories. In addition, the website provides access to a database where planned and scheduled observations are listed for all participating observers, to enable real-time coordination while observing. This website is password protected. To gain access to the website, please email Stef McLaughlin stefmcl@astro.umd.edu.

\subsection{Encounter Science from Earth}

Key Earth-based observations at the time of encounter include both wavelength and timescale regimes which are inaccessible to the Deep Impact spacecraft, and in particular to observe the development of any long-term activity changes which may occur as a result of the impact. Table 8 summarizes the key science goals from Earth at encounter, and the wavelength regimes for observations.

\subsection{Discussion of the Worldwide Plans}

Many of the national and international observatories had an open competition for observations at the time of impact, rather than a dedicated observing campaign. However, a few observatories have declared the night of impact as director's discretionary time (e.g. Keck, Subaru, Gemini), and the NASA IRTF has a long dedicated campaign. Below we discuss the various strategies and approaches that will be utilized for National and International observing facilities as well as the time that has been already allocated. Figure 5 summarizes these world wide plans for both the facilities which will directly view the encounter, and for those that will not. The primary Earthsupport science goals are listed, along with the observing technique and wavelength regime required to achieve these goals, and the telescope facilities and instrumentation best suited to achieving these 
goals are listed.

While not all facilities will be able to observe the actual moment of impact, the possible new activity could take hours or even days to fully develop, so observatories at all longitudes will play an extremely valuable role in understanding the scientific implications of the impact. Therefore for our purposes here we refer to the "encounter" as including the impact as well as many days afterward. Table 9 summarizes the allocation of telescope time at observatories world wide as known in January 2005.

At the time of the encounter, the observations will be coordinated with a control center at the summit of Mauna Kea (probably located in the NASA IRTF Facility). Telescopes will be connected via polycom connections to the IRTF control center, which will be connected via polycom to the mission operations at the Jet Propulsion Laboratory in Pasadena. This will ensure rapid and accurate dissemination of the events at encounter so that observers can revise observing strategies in real time.

\subsubsection{Mauna Kea and Haleakala Observatories}

The summit of Mauna Kea, Hawaii, will be the premier site for the real-time observations at encounter. There are 12 telescopes at the summit and in the sub-mm valley (counting the Smithsonian Array as a single telescope). Most of these facilities have multiple instruments which will be of use during the encounter. At most facilities, telescope time was awarded on the basis of open competition within the community. The Keck and IRTF TACs augmented their process with input from the Deep Impact science team. Gemini and Subaru are considering the three days of UT July 3 , 4, and 5 to be director's discretionary (DD) time. Keck is considering UT July 3 alone to be DD time.

The current slate of instrumentation scheduled for Mauna Kea telescopes (at time of writing, midJanuary 2005) is as follows.

- The Keck I and II telescopes will use HIRES and NIRSPEC, respectively, on UT July 4, 5 , and 6. HIRES will allow high-spectral resolution capability in the optical and near-UV (particularly crucial for isotopic work). NIRSPEC allows high resolution near-IR spec- troscopy to detect volatile organic parent molecules.

- With its closed loop adaptive optics system, the Gemini $8 \mathrm{~m}$ telescope is optimized for high spatial resolution in the near-IR. It is also equally well-suited to performing diffraction limited mid-IR observations; One optimal use of this facility would be to use it for 10 and $20 \mu \mathrm{m}$ spectroscopy using the Michelle instrument. This region is diagnostic of refractory silicate features which may trace the thermal history in the solar nebula.

- The UH2.2m telescope has a unique instrument that is permanently installed at the bent-cassegrain focus: the Supernova Integral Field Spectrograph (SNFS) which will allow us to obtain spatially resolved integral field spectroscopy over the 3000 to $10000 \AA$ range of the nucleus and inner coma of comet 9P/Tempel 1 before, during, and in the days after the impact. The spectral region covered by these data contains many molecular emission features that will be diagnostic of the temporal sequence of release of volatiles from the comet as a result of the impact. It may be possible to get adequate $\mathrm{S} / \mathrm{N}$ at impact time to get spectra with time resolution of 1 minute.

- In addition the UH2.2m will have a moderatefield $\left(\sim 7^{\prime}\right)$ CCD camera available for several nights before, during, and after the impact. The UH0.6m telescope will make use of an even wider field orthogonal-transfer CCD (Burke et al., 1994). This camera can read out CCD images with tip-tilt adaptive optics at video rate.

- The Subaru 8m telescope have several instruments that may reflect the optimal use of that facility. The Japanese community is strongly interested in utilizing two high speed high definition TV cameras, one to image the sky as visual observers will see the event.

- The UKIRT $3.8 \mathrm{~m}$ telescope will make use of its high-resolution infrared spectrometer, CGS4, on UT July 3, 4, and 5. Their priority is to observe the evolution of the "hot" 
bands of water before, during, and after the impact.

- The only instrument available at time of impact for the Canada-France-Hawaii $3.6 \mathrm{~m}$ telescope will be the wide field imager Megacam. Thus this facility will be well-suited for watching the development of the dust coma and tail, especially post-impact.

- The robotically-controlled Faulkes $2 \mathrm{~m}$ telescope atop Haleakala on Maui will be also observing before, during, and after the impact with a moderate-field $\left(\sim 4.6^{\prime}\right) \mathrm{CCD}$ camera.

- The Air Force's 3.6m AEOS telescope also atop Haleakala will be observing the impact with a high-resolution $(R \sim 17000$ to 49000$)$ visible spectrometer, covering the 6400 to $10000 \AA$ A region.

\subsubsection{European Southern Observatory}

At the Deep Impact workshop held in Garching, Germany, during February 2004, a number of European planetary scientists met and discussed optimal observing strategies for ESO. Two European teams were created to lead in the development of coordinated large proposals to ESO for Period 74 (pre impact) and 75 (encounter). Chile will not be able to see the comet until about 16 hours after impact but the observatories will certainly be able to follow the short-term evolution of the comet's behavior.

The nucleus structures and dust team, lead by H. Boehnhardt and U. Käufl, has been awarded the equivalent of 19 nights of time spread out over six telescopes at La Silla and Cerro Paranal around the time of impact. They will: perform high resolution near-IR imaging to look for ejecta and jets (VLT UT4 with NACO; NTT with SOFI); obtain mid-IR images and spectra to investigate dust composition, in particular silicate evolution (VLT UT3 with VISIR, $3.6 \mathrm{~m}$ with TIMMI2); observe the comet in optical wavelengths to characterize polarization (VLT UT1 with FORS1) and to study dynamical effects and scattering properties of the dust (2.2m with WFI, NTT with EMMI). Having complementary programs at both Cerro Paranal and La Silla will ensure some backup in case of poor weather.
The team studying the evolution of the gas coma, lead by H. Rauer, had been awarded the equivalent of 8 nights of time at Paranal for optical and near-UV spectroscopy. High dispersion spectroscopy will be used to investigate isotopic ratios (VLT UT2 with UVES), and low dispersion spectroscopy will be used to get pre- and postabundances of molecular species such as $\mathrm{CN}, \mathrm{C}_{2}$, $\mathrm{C}_{3}$ etc. (VLT UT1 with FORS2).

\subsubsection{National Optical Astronomy Observato- ries}

Access to the primary facilities of the National Optical Astronomy Observatories (NOAO) on Kitt Peak and Cerro Tololo was via open competition through the normal proposal process. Time at other facilities where the US astronomical community has access through NOAO was generally likewise determined via open competition. However, the Deep Impact worked closely with the astronomical community to identify the areas where NOAO can make the greatest contribution.

The telescopes at Kitt Peak will be able to see the impact in real time, however, the comet will be at very high $(\chi>2.5)$ airmass. The moderate to large telescopes there - the Mayall $4 \mathrm{~m}$, the WIYN $3.5 \mathrm{~m}$, and the $2.1 \mathrm{~m}$ telescopes - have been allocated for $9 \mathrm{P} /$ Tempel 1 observations around the time of encounter. At the Mayall, the MOSAIC instrument will be used to obtain narrowband imaging and photometry. At the $2.1 \mathrm{~m}$, the near-IR camera SQIID will give us a unique capability that is not available anywhere else: truly simultaneous photometric imaging in JHK (and possibly L, if weather cooperates). At the WIYN, we will be using an integral-field unit "Densepak" to obtain visible spectroscopy simultaneously at many different locations in the coma.

At Cerro Tololo, the Blanco 4m telescope has been allocated to observe the comet using ISPI, a large-format near-IR camera. ISPI is the largest near-IR imager in South America, and would therefore make a unique contribution. Among other moderate and small telescopes at CTIO, we have been allocated time at the $0.9 \mathrm{~m}$ and the $1.5 \mathrm{~m}$ telescopes, which are run by the SMARTS consortium. At the $0.9 \mathrm{~m}$, a classically-scheduled run using CFCCD, a 2K-by-2K CCD camera, has been scheduled. At the $1.5 \mathrm{~m}$, we will use CPAPIR, another wide-field large-format near-IR camera. 
With these three telescopes at CTIO, we have a capability not found elsewhere: the ability to obtain deep simultaneous images of the dust from U-band through K-band. Combined with dust dynamical modelling, thermal data planned from Spitzer and other telescopes, there is the potential to thoroughly characterize the dust component and to search for differences between the preimpact dust (from the more evolved comet surface layers) and the post-impact dust excavated from the interior.

The SOAR $4 \mathrm{~m}$ and Gemini-South $8.2 \mathrm{~m}$ telescopes are located on Cerro Pachón. A possible optimal scenario that would complement the other observations being made from Mauna Kea at impact time is to use PHOENIX, a high resolution $(R=50,000)$ IR echelle spectrograph on SOAR to look for organics, and use the TReCS $10-20 \mu \mathrm{m}$ spectrograph on Gemini to monitor the chemical and silicate evolution in the mid-IR.

\subsubsection{Lowell Observatory}

Lowell Observatory is a privately owned astronomical research institution located in Flagstaff Arizona. The observatory has 3 telescopes available at Anderson Mesa for research projects: the 1.8-m Perkins telescope, the 42-inch Hall telescope, and the 31-inch robotically operated telescope. The $1.8-\mathrm{m}$ has optical imaging and spectroscopic capabilities as well as a newly commissioned near-IR imaging polarimetric spectrograph, Mimir. Mimir utilizes an Alladin III InSb $1024 \times 1024$ dectector which operates between 1 $5 \mu \mathrm{m}$. It has two cameras providing a choice of plate scale of either $0.6 \operatorname{arcsec}_{\text {pix }}{ }^{-1}$ or $0.18 \operatorname{arcsec}$ $\mathrm{pix}^{-1}$. There are four filter wheels that provide a variety of functions including standard bandpass filters (i.e., JHKLM), polarimetric elements $(H$ band only), and grisms for spectroscopy at resolving powers over a range from 20 to 2000 . The wide field camera with a grism employs 5 arcminute slits from 1 to 10 pixels wide. This may make a unique contribution to the real-time encounter observations in that the system will be capable of obtaining high time resolution near-IR spectra which may be able to study the impact flash. The Hall telescope may be used with the KRON photometry or the SITE $2 \mathrm{~K}$ CCD for narrow band photometry or imaging of the coma to look at the production rates of various gaseous species. The 31-inch robotic telescope will play a critical role in monitoring the development of the dust coma on a nightly basis, weather permitting, starting early in the spring 2005, through encounter until after the impact when the comet moves into solar conjunction. This will be useful to watch for brightness fluctuations and outbursts in activity, and search for jets in the inner coma, and after the upgrade to a large format CCD, to obtain images for dust dynamical modelling.

\subsubsection{Palomar Observatory}

The Palomar Observatory located in California, is operated by the California Institute of Technology. Facilities include the Hale 200-inch telescope, the 60-inch, Oshin 48-inch, and the 18-inch schmidt telescopes. The 200 -inch telescope will be utlized with the adaptive optics system and nearIR imaging to watch the evolution of the dust that is ejected during impact, and to look for evidence of water-ice in the near IR as the crater is excavated.

\subsubsection{Lick Observatory}

The University of California Observatories operates Lick Observatory on Mt. Hamilton, CA for the University of CA community. The observatory has 3 major telescopes: the 120-inch Shane telescope, the Coude Aux Telescope and the Nickel 40 -inch reflector, in addition to 5 smaller telescopes. The Shane telescope has been scheduled for 5 nights at the time of impact with a visitor instrument that can perform 0.5-2.5 micron lowresolution spectroscopy. The goal will be to monitor the evolution of the impact flash and development of activity in the gas and dust post-impact. The smaller telescopes are not yet scheduled for the encounter period, but plans are underway to utilize the 40-inch for imaging.

\subsubsection{Las Campanas Observatory}

The Carnegie Institution of Washington operates facilities at the Cerro Las Campanas observatory in Chile. The observatory facilities include the twin 6.5-m Magellan telescopes (Baade and Clay), the du Pont 2.5-m telescope and the Swope $1.0-\mathrm{m}$ telescope. Near-IR and visible imaging is scheduled at Magellan (using PANIC the 1024 IR imager on Baade) two weeks prior to the encounter 
in order to establish baseline observations in collaboration with a thermal-IR project at Gemini south. Although the Magellan telescopes are currently only scheduled through June 27, 2005, the plan is to observe through encounter and the postencounter period, monitoring the comet for 8 rotation periods to monitor the freshly excavated active area and the evolution of the particles in the coma. Imaging and spectroscopy are scheduled at the $2.5-\mathrm{m}$ duPont and 1.0-m Swope telescopes throughout the encounter period.

\subsubsection{Radio Facilities}

Ground based facilities where observations at sub-millimeter and/or millimeter wavelengths are planned and/or already scheduled include:

- the IRAM 30m telescope in Spain, which can observe simultaneously 4 lines between 86 and $272 \mathrm{GHz}$;

- the IRAM Plateau de Bure interferometer in France, which has six $15 \mathrm{~m}$ telescopes and can observe at $86-115 \mathrm{GHz}$ and at $205-250$ $\mathrm{GHz}$ simultaneously (although autocorrelation mode will likely be more efficient to make detections than interferometric mode, thus reducing the interest of interferometers for such a modest activity comet);

- the $15 \mathrm{~m}$ JCMT and 10m CSO atop Mauna Kea, which have receivers available at 210-275 GHz, 300-365 GHz (330-365 for JCMT), and 450-510 GHz;

- the APEX $12 \mathrm{~m}$ test antenna in Chajnantor (5000m elev.), Chile (pending availability of heterodyne receivers);

- the Kitt Peak $12 \mathrm{~m}$ antenna (pending availability of $260 \mathrm{GHz}$ receiver);

- the SMA, atop Mauna Kea, which consists of eight $6 \mathrm{~m}$ antennas that observe in the 230 and $345 \mathrm{GHz}$ bands;

- the $100 \mathrm{~m}$ GBT in West Virginia, which can observe at a range of bands at millimeter, centimeter, and decimeter wavelengths;

- the Australian facilities, consisting of the Parkes $64 \mathrm{~m}$ (operating between 10-70cm, up to $24 \mathrm{GHz}$ ) which can access lines of $\mathrm{H}, \mathrm{OH}$,
$\mathrm{CH}_{3} \mathrm{OH}$, and $\mathrm{CH}_{4}$, among others; the Australian Telescope Compact Array which operates between $85-105 \mathrm{GHz}$ in the period from April through October; and the Mopra $22 \mathrm{~m}$ (operating at $3,6,13$, and $20 \mathrm{~cm}$, and 3 and $12 \mathrm{~mm}$ );

- the Very Large Array in Socorro, New Mexico, which consists of 2725 -meter dishes and can observe between 74 to $50,000 \mathrm{MHz}$ (400 to $0.7 \mathrm{~cm}$ ); and

- other radio-telescopes (e.g., Nobeyama 45m, FCRAO $14 \mathrm{~m}$ ) that can observe around 89 $\mathrm{GHz}$, in which lies the $\mathrm{HCN}(1-0)$ line that is somewhat harder to detect than the $\mathrm{HCN}(3-$ 2) line.

Note that CARMA (the BIMA+OVRO merged array) will not be ready in time, and that the comet's declination is unreachable from Arecibo.

The sub-mm fundamental line of $\mathrm{H}_{2} \mathrm{O}$ at 557 $\mathrm{GHz}$ will be observable from space observatories such as ODIN. The ODIN satellite is a Swedish satellite with a $1 \mathrm{~m}$ antenna, which has receivers at $119 \mathrm{GHz}$ and four in the sub-mm band (480-580 $\mathrm{GHz}$ ). The pointing constraints are such that the comet will be observable only between $60-120^{\circ}$ solar elongation (June-Sep. 2005). In addition, due to its low earth orbit, the comet will be only visible for 60 minutes every 96 minute orbit. Observing constraints for the SWAS satellite, also operating around $557 \mathrm{GHz}$, are similar: elongation $>75^{\circ}$ (comet 9P/Tempel 1 will thus be visible most of the year 2005) but also about $1 \mathrm{hr}$ per $1.6 \mathrm{hr}$ orbit. While this would be within the capabilities of the SWAS satellite, it is not scheduled to be operational at this time (out of funding).

\subsubsection{Other Efforts}

Chinese astronomers at the National Central University in Taiwan are coordinating a large number of far eastern and eastern European observatories. Located at dark sites, these will fill in the gap in longitude coverage for this part of the world. The instrumentation at these facilities will be ideally suited to optical imaging and polarimetry of the dust. The observatories include Moletai (Lithuania), Gaumeigu (China), Yunnan (China), Lulin (Taiwan) and Majdanak (Uzbekistan). 
A consortium of astronomers plans to utilize the facilities on La Palma both for extensive monitoring of the comet during the spring 2005 both for weekly monitoring of the development of the dust coma, and to monitor the onset of molecular gas production (low resolution optical spectra). At encounter, there are plans for doing near IR spectroscopy to look at organic species and high resolution JHK near-IR imaging.

In addition to participation through the Subaru telescope on Mauna Kea, the Japanese government has provided funds to build a new 1-m telescope on Ishigake Island (in the Okinawa Islands, just north of Taiwan). Deep Impact is the driver for completion and science readiness by July 2005. The first light instrument will be PICO, and optical polarimetric imager for dust observations.

Observers in Russia are planning to use the $6 \mathrm{~m}$ SAO telescope, the $2.0 \mathrm{~m}$ telescope, and the $2.6 \mathrm{~m}$ telescopes for both imaging and medium and high resolution spectroscopy (optical) leading up to the encounter and at the time of encounter. Colleagues in Bulgaria will perform complementary narrow and wide band imaging of the dust coma of the comet.

At the Anglo-Australian Observatory, several nights around the time of impact have been allocated. The AAT will use UCLES, a visible-wavelength echelle spectrograph. The UK Schmidt Telescope will use 6DF, a multi-object visible-wavelength spectrograph with an enormous six-degree field of view.

At Calar Alto Observatory, the $2.2 \mathrm{~m}$ telescope will be used to monitor the comet with CAFOS, a visible-wavelength instrument capable of obtaining images and long-slit spectra. From early January 2005 through July 1, 2005, broadband CCD images will be obtained of the comet every 2-3 days. Once a month spectra will be obtained to watch the development of the gas species, and an additional focussed run for both imaging and spectroscopy is scheduled for April 2005.

At Fort Davis, the optimal use of the $9.2 \mathrm{~m}$ Hobby-Eberly Telescope will be to obtain highspectral resolution monitoring observations of the comet over the course of 2005 through June.

At the Observatorio del Roque de los Muchachos, on La Palma, the $4.2 \mathrm{~m}$ WHT has been scheduled to use OASIS, an integral-field visible- wavelength spectrograph combined with $\mathrm{AO}$, and LIRIS, a moderate-resolution near-IR spectrograph. At the $3.6 \mathrm{~m}$ TNG, three instruments will be used: DOLORES, a moderate-field CCD imager; SARG, a high-resolution visible spectrograph; and NICS, a near-infrared camera. Finally, at the $2.5 \mathrm{~m}$ NOT, the plan is to use ALFOSC, a faint-object visible-wavelength spectrograph.

The PLANET consortium of telescopes is a global network for monitoring gravitational microlensing events to search for extrasolar planets. This consortium will be obtaining nightly R-band observations of the comet starting in early spring 2005, through the post-encounter period, primarily using the 1.5-m Boyden observatory in South Africa, and the 1-m Canopus telescope in Hobart Tasmania. Back up observatories in case of poor weather include the 2-m Pic du Midi telescope and the 1.5-m Danish telescope at La Silla. In addition, the consortium will be obtaining weekly BVRI color images.

\subsubsection{Unique Facilities and Special Arrange- ments}

Hubble Space Telescope - Cycle 13 time has been awarded to the Deep Impact team and collaborators to study the generation and evolution of the gaseous coma resulting from the impact. Time was awarded to obtain ultraviolet spectra with STIS before, during and after impact, with the goal of detecting highly volatile species such as $\mathrm{CO}, \mathrm{CO}_{2}$ and $\mathrm{S}_{2}$ from newly exposed sub-surface layers. However, with the loss of the STIS instrument after the suspend state on 2004 August 3, a subset of these observations will probably be made with the ACS. In addition, we will be using the high resolution capabilities of the ACS/HRC in conjunction with the camera aboard the Deep Impact spacecraft two weeks prior to impact to use stereo imaging to determine the spatial orientation of active jets emanating from the comet's nucleus and to assess potential hazards of these jets to the spacecraft.

It will be possible to time the impact so that we guarantee that HST will be able to observe the comet at that exact moment, but given the uncertainties in the telescope's orbital dynamics, the exact moment of impact will not be decided until several weeks before UT July 4. 
GALEX Ultraviolet Telescope - The Galaxy Evolution Explorer is an explorer class 0.5-m orbiting ultraviolet telescope launched 28 April 2003 for a 29 month mission. The instruments are

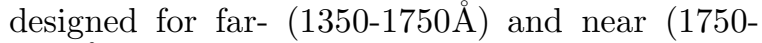
$2800 \AA$ ) UV imaging and spectroscopy (resolution: $\left.\mathrm{R}_{F U V}=250-300, \mathrm{R}_{N U V}=80-150\right)$. Deep Impact team members and collaborators have received GALEX telescope time to study the changes in the gas coma (in particular $\mathrm{CO}$ and $\mathrm{CO}_{2}$ ) resulting from the excavation of subsurface material due to impact. GALEX cannot observe HI, OI or $\mathrm{OH}$ which are the principal dissociation products of $\mathrm{H}_{2} \mathrm{O}$, but it will be able to observe emissions of CI and the CO Fourth Positive system and the CO Cameron band system. The latter arises from prompt emission following photodissociation of $\mathrm{CO}_{2}$, and can therefore provide a measure of $\mathrm{CO}_{2}$ abundance. $\mathrm{CO}_{2}$ cannot be measured from the ground in the infra-red. Changes in cometary activity from the quiescent state will be monitored using emission from the $(0,0)$ band of CS at $2576 \AA$. The parent of CS $\left(\mathrm{CS}_{2}\right)$ photodissociates very rapidly to $\mathrm{CS}$, so the $\mathrm{CS}$ abundance tracks the changes in outgassing with very little phase lag. Because of a strong g-factor compared to $\mathrm{CO}$, this molecule is relatively easy to detect.

FUSE Observatory - The Far Ultraviolet Spectroscopic Explorer is a high-orbit extreme UV space observatory operating in the range 905$1195 \AA$ with a spectral resolution of $15 \mathrm{~km} \mathrm{~s}^{-1}$ (i.e. about $0.05 \AA$ ). Several reaction wheels on the spacecraft are non-functional, so there are severe restrictions on the spacecraft pointing, but there are several important scientific objectives that can be addressed if some of the restrictions can be relaxed and the observatory pointed at 9P/Tempel 1. In particular the loss of the STIS instrument on HST leaves FUSE as the most capable facility for several items. A review of ultraviolet observations of cometary comae and the important questions is given by Feldman et al. (2005). FUSE can:

- search for argon emission and O VI emission after impact. An unconfirmed possible detection of argon has only been made in comet C/1995 O1 (Hale-Bopp). The argon abundance is dependent on the temperature environment at which the comet's ices con- densed (Weaver et al. 2002; Notesco et al. 2003).

- provide a very sensitive probe of $\mathrm{CO}$ before and after the impact. While $\mathrm{CO}$ is commonly seen in long-period comets, an assessment of the remaining abundance within a highly-evolved short-period comet (which has never been done) would give clues to the thermal and structural history of the nucleus.

- possibly detect $\mathrm{N}_{2}$ for the first time in a comet $(958.6 \AA)$. Among nitrogen compounds this species should have been dominant in the solar nebule (Feldman et al. 2005) so a measurement of its abundance in comets would be fruitful.

- observe $\mathrm{H}_{2}$ emission before and after the impact and watch its temporal variation. Detection of the emission (near $1600 \AA$ ) requires high spectral resolution. One important question is determining if there is any nuclear source of $\mathrm{H}_{2}$ in addition to the extended source of dissociated $\mathrm{H}_{2} \mathrm{O}$.

- possibly observe the $\mathrm{D} / \mathrm{H}$ ratio post-impact, which would be the first such measurement in a Jupiter-family comet, and hence could be a probe of $\mathrm{D} / \mathrm{H}$ in a different region of the solar nebula.

- monitoring the abundances of atomic C, O, $\mathrm{H}, \mathrm{S}$ and $\mathrm{N}$ in the coma before and after impact. In previous FUSE observations of comets, over 90 emission lines have been detected, but over half of these have yet to be identified. Because the FUSE bandpass covers the resonance transitions of nearly all atomic elements and many simple ions, there may be a rich set of post-impact observations.

Chandra X-Ray Telescope and the XMM Newton Observatory - In the X-ray regime, the Deep Impact experiment allows for a controlled test of the charge exchange (CXE) emission mechanism that drives cometary X-ray emission (Lisse et al. 2001; Kharchenko \& Dalgarno 2001; Krasnopolsky et al. 2002). Previous ROSAT 
and Chandra observations studied cometary Xray emission as the solar wind changed but the cometary emission remained constant. Here at a precise time, a fresh amount of neutral material will be injected into a finite volume of the extended cometary coma. This new material will directly increase the emission measure for the comet, passing from the collisionally thick to the collisionally thin regions of emission over the course of days. The DI experiment also allows for a direct search for prompt X-rays created by hyper-velocity impact processes.

The Deep Impact team has received $300 \mathrm{ksec}$ of Chandra time to observe 9P/Tempel 1 before, during and after the impact encounter. Pre-impact, we expect a modest X-ray signal from the comet from the interaction of its neutral gas coma with the $10^{6} \mathrm{~K}$ solar wind plasma through the charge exchange mechanism. At encounter, the impactor vaporizes within $1 \mathrm{msec}$ and is blown back out in a plume of vapor and plasma. Hypervelocity impact experiments at NASA Ames (Sugita et al. 2003) suggest the prompt production of abundant ionized and atomic species, strong magnetic fields and initial plasma temperatures of $\sim 10^{6} \mathrm{~K}$. There may be a rapid non-equilibrium creation of an expanding plasma cloud with possible short-term Xray generation. At Encounter, Chandra will spectroscopically observe the creation and evolution of the impact plume. Post-impact, the X-ray luminosity should increase 30 -fold. The total integrated X-ray luminosity increase will be used as one of the best estimates of the total neutral gas mass ejected due to the impact. The differences in the optical and X-ray light curves will be used to probe the extent of the collisionally thick X-ray emitting region around the nucleus.

The X-ray Multi-Mirror (XMM) satellite is an ESA orbial X-ray observatory. XMM has 3 instruments which are effective over the energy range from $300 \mathrm{eV}$ to $12 \mathrm{keV}$ : EPIC which performs spatially resolved moderate resolution X-ray spectroscopy, RGS which perfoms high-spectral resolution soft X-ray spectroscopy and OM which can obtain optical and UV images below $5000 \AA$. While complementary, Chandra will not be able to get as good spectral quality data as XMM-Newton, and indeed only RGS can separate OVII and OVIII. However, time was not awarded on this facility.
SOHO Observatory - The Solar and Heliospheric Observatory ( $\mathrm{SOHO}$ ) is an international project to study the sun. Because it points at the Sun at all times, it will not be possible to use the LASCO imager or UVCS spectrometer to look at the comet, however, the observatory has an all-sky Lyman- $\alpha$ imager, SWAN (the Solar Wind Anisotropy Monitor) which could be used to track the comet's emission over time and orbit. The SOHO solar wind data will be important to understand the solar wind environment at the comet during the encounter. This will help us to understand the X-ray emission we see. This emission is dominated by lines of ionized and excited carbon, oxygen, sometimes neon, and possibly nitrogen.

Spitzer Space Telescope - Spitzer Space Telescope observations of comet 9P/Tempel 1 are possible at the end of Cycle 1 , in a viewing window that starts 2 days before the encounter, ending 3 months later, and time has been awarded primarily for spectroscopy but also for imaging with the IRS instrument. Although there were many possibilities for the use of the Spitzer telescope, for observations at the exact time of impact the Deep Impact team made the decision to utilize the unique capabilities of the telescope in a wavelength regime which is largely inaccessible from the ground: the $5-8.7 \mu \mathrm{m}$ window. The IRS data in combination with high-resolution spectroscopy from the spacecraft itself will let us measure the spectral energy distribution from 1 to $38 \mu \mathrm{m}$.

The IRS's wavelength region has heretofore been very poorly explored for comets because of the high terrestrial atmospheric opacity. There are a wealth of organic species that may be detected, including $\mathrm{HCN}, \mathrm{CS}_{2}, \mathrm{CH}_{4}, \mathrm{CH}_{3} \mathrm{OH}$, $\mathrm{CH}_{2} \mathrm{O}, \mathrm{CH}_{3} \mathrm{OCH}_{3}, \mathrm{H}_{2} \mathrm{O}, \mathrm{NH}_{3}, \mathrm{CH}_{3} \mathrm{NH}_{2}, \mathrm{C}_{2} \mathrm{H}_{6}$, $\mathrm{C}_{2} \mathrm{H}_{2}$ and $\mathrm{C}_{2} \mathrm{H}_{5} \mathrm{OH}$. The neutral gas species will give us clues to the difference between surface and subsurface composition. For tens of seconds the plume could be hot enough for us to detect polycyclic aromatic hydrocarbons (PAHs) with features at $6.2,7.7$, and $8.6 \mu \mathrm{m}$, which have never been seen before in comets. We will search for the $6.85 \mu \mathrm{m}$ solid state feature seen in dense molecular clouds, which may be due to a $\mathrm{C}-\mathrm{H}$ deformation in hydrocarbon-containing ices or caused by carbonate materials. Lastly we will also monitor the 8-13 $\mu \mathrm{m}$ and $18 \mu \mathrm{m}$ dust silicate features for changes in 
the degree of crystallinity. All these phenomena carry interesting potential diagnostics of cometary composition and formation.

We have requested continuous monitoring from the start of the encounter window through 2 days post-impact to observe the immediate effects of the impact. There will also be brief visits 5, 10, 20,50 and 100 days post-impact to follow the longterm evolution of the comet.

In addition, we have been awarded time in the months leading up to the encounter to characterize the dust from the comet in the 8-38 $\mu \mathrm{m}$ window. Among other goals, this will let us determine the spacecraft hazard from large dust in the near-nucleus environment. Modeling of the dust spectral energy distribution will tell us the emissivity, grain size distribution, and mass of the large grains.

Our pre-encounter program also involves deep imaging at 24, 70, and $160 \mu \mathrm{m}$ with the MIPS instrument to characterize the comet's dust trail.

NASA Infra-Red Telescope - NASA's IRTF will have an extended observing campaign of the comet in June-July 2005. Seven instruments - NSFCAM, MIRSI, SpeX, BASS, HIFOGS, CShell, and the new Apogee CCD camera - will be in use at various times from late June through early $\mathrm{Au}-$ gust, acquiring data for five PI programs. On the actual impact day, SpeX is expected to be in use, taking high frame-rate observations in the nearIR. Observations will usually be done by an support astronomer, with cometary scientist (or scientists) frequently on site. Because of the unpredictable nature of the event, and because of the need for coordination with the world-wide ground based observing campaign, the projects will be observed in queue mode, and the behavior of the comet after impact will dictate what is actually observed. The proprietary period for the data is 6 months, and the IRTF will provide PDS-compliant FITS headers, and will make the DI data archive available to the public.

Rosetta Spacecraft - The Rosetta spacecraft will be in a slightly better viewing condition than Earth-orbital satellites (comet geocentric distance $0.53 \mathrm{AU}$ versus $0.89 \mathrm{AU}$ in early July 2005) and the MIRO $0.3 \mathrm{~m}$ sub-mm telescope, although less sensitive than other Earth satellites, can be in permanent viewing of the comet at that time, to monitor the $557 \mathrm{GHz} \mathrm{H}_{2} \mathrm{O}$ line. Rosetta can also offer a range of observing techniques for observing the impact, using OSIRIS for imaging, VIRTIS for near-IR spectra and ALICE for far UV spectra. The ALICE instrument on Rosetta does some of the same things as FUSE, albeit with lower sensitivity. The plan for Rosetta will be to begin observing the comet one week before the encounter and for two weeks after.

Sofia Telescope - The Sofia airborne observatory is an infrared-optimized telescope which is flown in the back of a Boeing 747 airplane. While the facility will not be flight ready in time for Deep Impact, there is the possibility that the telescope could be used on the ground, assuming the airplane can be moved from Waco TX, where the comet will be below the horizon at impact, and moved to NASA Ames. With the HIPO occultation instrument, wit will be possible to get simultaneous $\mathrm{V}$ and $\mathrm{R}$ observations with $20 \mathrm{~Hz}$ time resolution and $\mathrm{S} / \mathrm{N} \sim 100$.

\section{Post-Encounter Observations}

The comet will be observable into early September 2005. During the time from impact into September, there should be relatively frequent monitoring of the gas production and dust evolution in order to fully assess the long-term changes induced by the impact. National facilities where longer term queue scheduled observations are possible, and private observatories such at the University of Hawaii $2.2 \mathrm{~m}$ telescope on Mauna Kea, will be ideally suited for this sort of monitoring.

Support for this work was provided through University of Maryland and University of Hawaii subcontract Z667702, which was awarded under prime contract NASW-00004 from NASA.

\section{REFERENCES}

A'Hearn, M. F., R. L. Millis, D. G. Schleicher, D. J. Osip and P. V. Birch (1995). The ensemble properties of comets: results from narrowband photometry of 85 comets, Icarus 118, 223-260.

A'Hearn, M. F., M. J. S. Belton, A. Delamere and 
W. H. Blume (2005). Deep Impact: A largescale active experiment on a cometary nucleus. Space Science Reviews this volume.

Belton, M. J. S, K. J. Meech, M. F. A'Hearn, O. Groussin, L. McFadden, C. Lisse, Y. Fernández, J. Pittichová, H. Hsieh, J. Kissel, K. Klaasen, P. Lamy, D. Prialnik, J. Sunshine, P. Thomas and I. Toth (2005). Deep Impact: Working properties for the target nucleus - Comet 9P/Tempel 1, Space Science Reviews this volume.

Biver, N. D., Bockelée-Morvan, J. Crovisier, P. Colom, F. Henry, R. Moreno, G. Paubert, D. Despois, D. C. Lis (2002). Chemical composition diversity among 24 comets observed at radio wvelengths. Earth Moon Planets, 90, 323333.

Blume, W. H. (2005). Deep Impact: Mission design, Space Science Reviews, this volume.

Buratti, B. J., D. M. Hicks, L. A. Soderblom, D. Britt, J. Oberst and J. K. Hillier (2004). Deep Space 1 photometry of the nucleus of comet 19P/Borrelly. Icarus 167, 16-29.

Burke, B. E., R. K. Reich, E. D. Savoye and J. L. Tonry (1994). An Orthogonal-Transfer CCD Imager, IEEE Trans. Electron. Devices 41, 2482.

Delahodde, C. E., K. J. Meech, O. R. Hainaut, E. Dotto (2001). Detailed phase function of comet 28P/Neujmin 1, Astron. Astrophys. 376, 672685 .

Feldman, P. D., A. L. Cochran, and M. R. Combi (2005). Spectroscopic investigations of fragment species in the coma, in Comets II, eds. M. Festou et al., Univ. of AZ, in press.

Fernández, Y. R., C. M. Lisse, U. Kaufl, H. Peschke, B. Sibylle, H. A. Weaver, M. F. A'Hearn, Ph. Lamy, T. A. Livengood and T. Kostiuk (2000). Physical properties of the nucleus of comet 2P/Encke, Icarus 147, 145-160.

Fernández, Y. R, K. J. Meech, C. M. Lisse, M. F. A'Hearn, J. Pittichová, and M. J. S. Belton (2003). The nucleus of Deep Impact target Comet 9P/Tempel 1. Icarus, 164, 481-491.
Finson, M. L., R. F. Probstein (1968). A theory of dust comets. I. Model and equations. ApJ, 154, 327-380.

Ishiguro, M., J. Watanabe, F. Usui, T. Tanigawa, D. Kinoshita, J. Suzuki, R. Nakamura, M. Ueno, and T. Mukai (2002). First detection of an optical dust trail along the orbit of 22P/Kopff, ApJ 572, L117-L120.

Ishiguro, M., S. M. Kwon, Y. Sarugaku, S. Hasegawa, F. Usui, S. Nishiura, Y. Nakada and H. Yano (2003). Discovery of the dust trail of the Stardust comet sample return mission target: 81P/Wild 2. Ap J. 598, L117-L120.

Kharchenko, V. and A. Dalgarno (2001). Variability of cometary X-Ray Emission induced by solar wind ions, ApJ 554, L99-L102.

Krasnopolsky, V. A, D. J. Christian, V. Kharchenko, A. Dalgarno, S. J. Wolk, C. M. Lisse and S. A. Stern (2002). X-Ray Emission from Comet McNaught-Hartley (C/1999 T1), Icarus 160, 437-447.

Lebofsky, L. A. and J. R. Spencer (1989). Radiometry and thermal modeling of asteroids, in $\mathrm{As}$ teroids II, eds. R. P. Binzel, T. Gehrels and M. S. Matthews, Univ. AZ Press, Tucson, 128-147.

Lisse, C. M., D. J. Christian, K. Dennerl, K. J. Meech, R. Petre, H. A. Weaver and S. J. Wolk (2001). Charge Exchange-Induced X-Ray Emission from Comet C/1999 S4 (LINEAR), Science 292, 1343-1348.

Lisse, C. M, M. F. A'Hearn, T. L. Farnham, O. Groussin, K. J. Meech, U. Fink, and D. G. Schleicher (2005). The coma of comet 9P/Tempel 1, Space Science Reviews this volume.

Magnusson, P., M. A. Barucci, J. Drummon, K. Lumme, S. J. Ostro, J. Surdej, R. Taylor and V. Zappala (1989). Determination of pole orientations and shapes of asteroids, in Asteroids II, eds. R. P. Binzel, T. Gehrels, and M. S. Matthews, Univ. AZ Press, 66-98.

McFadden, L., M. Roundtree-Brown, E. Warner, S. McLaughlin, J. Behne, J. Ristvey, D. Duncan, S. Gillam and G. Walker (2005). Education and public outreach for NASA's Deep Impact 
Discovery mission. Space Science Reviews this volume.

Meech, K. J. and J. Svoreň (2005). Physical and Chemical Evolution of Cometary Nuclei, in Comets II, eds. M. Festou et al., Univ. of AZ, in press.

Meech, K. J., O. R. Hainaut, and B. G. Marsden (2004). Comet nucleus size distributions from HST and Keck telescopes. Icarus 170 463-491.

Noll, K. S., M. A. McGrath, L. M. Trafton, S. K. Atreya, J. J. Caldwell, H. A. Weaver, R. V. Yelle, C. Barnet, and S. Edgington (1995). HST spectroscopic observations of Jupiter after the collision of comet Shoemaker-Levy 9. Science 267, 1307.

Notesco, G., A. Bar-Nun, and T. Owen (2003). Gas trapping in water ice at very low deposition rates and implications for comets. Icarus $\mathbf{1 6 2}$, 183-189.

Sugita, S., P. H. Schultz and S. Hasegawa (2003). Intensities of atomic lines and molecular bands observed in impact-induced luminescence, JGR 108, 5140 .

Sykes, M. V. and R. G. Walker (1992). Cometary Dust Trails I - Survey, Icarus 95, 180-210.

Weaver, H. A., P. D. Feldman, M. R. Combi, V. Krasnopolsky, C. M. Lisse, and D. E. Shemansky (2002). A search for argon and O VI in three comets using the Far Ultraviolet Spectroscopic Explorer, Ap. J. 576, L95-L98.

Yeomans, D., J. Giorgini and S. R. Chesley (2005). The history and dynamics of comet 9P/Tempel 1, Space Science Reviews, this volume.

This 2-column preprint was prepared with the AAS LATEX macros v5.2. 


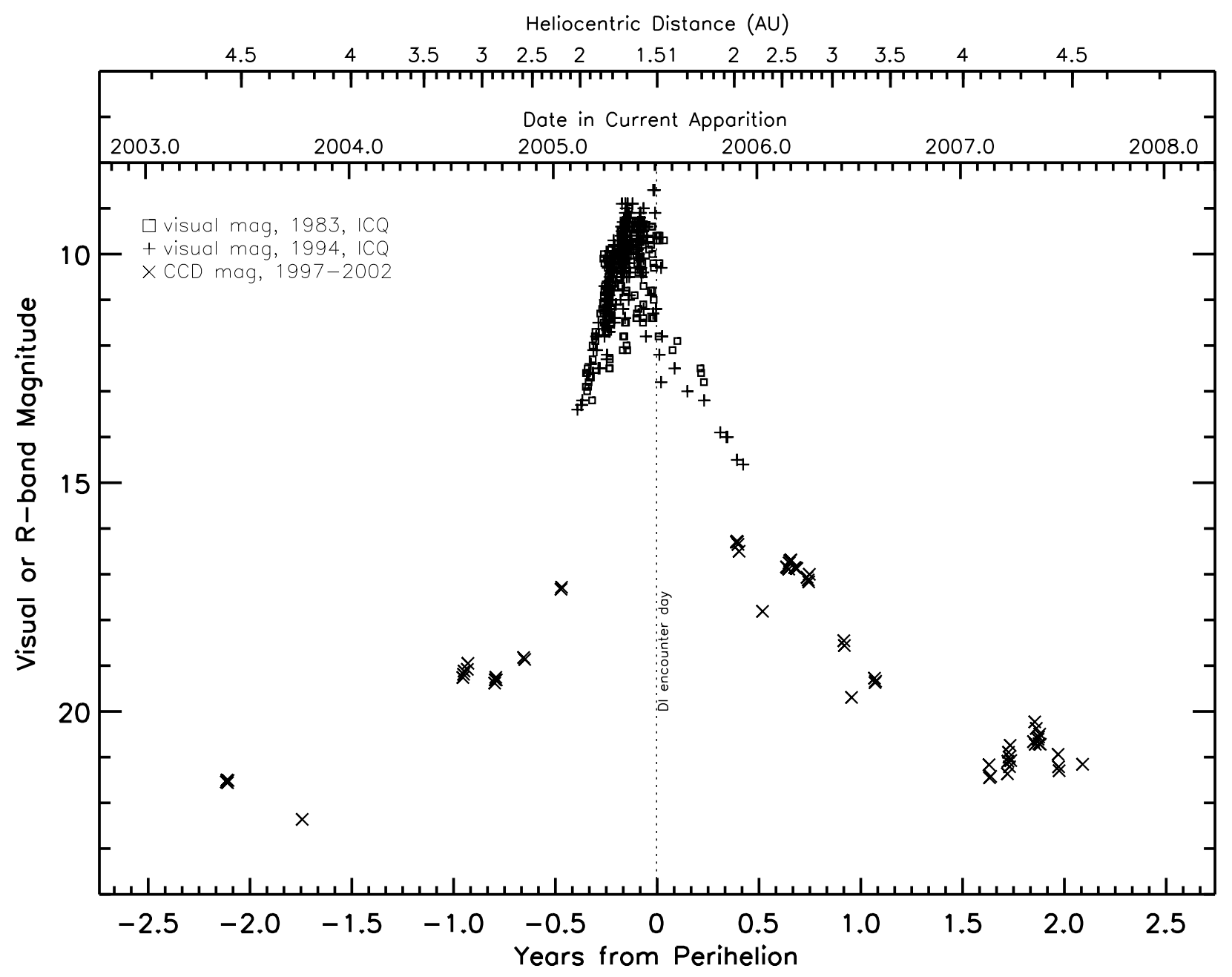

Fig. 1.- Light curve of comet 9P/Tempel 1 combining data from the ground-based program since 1999 and data from the $I C Q$. Note that the brightness peaks about 2 months pre-perihelion. 

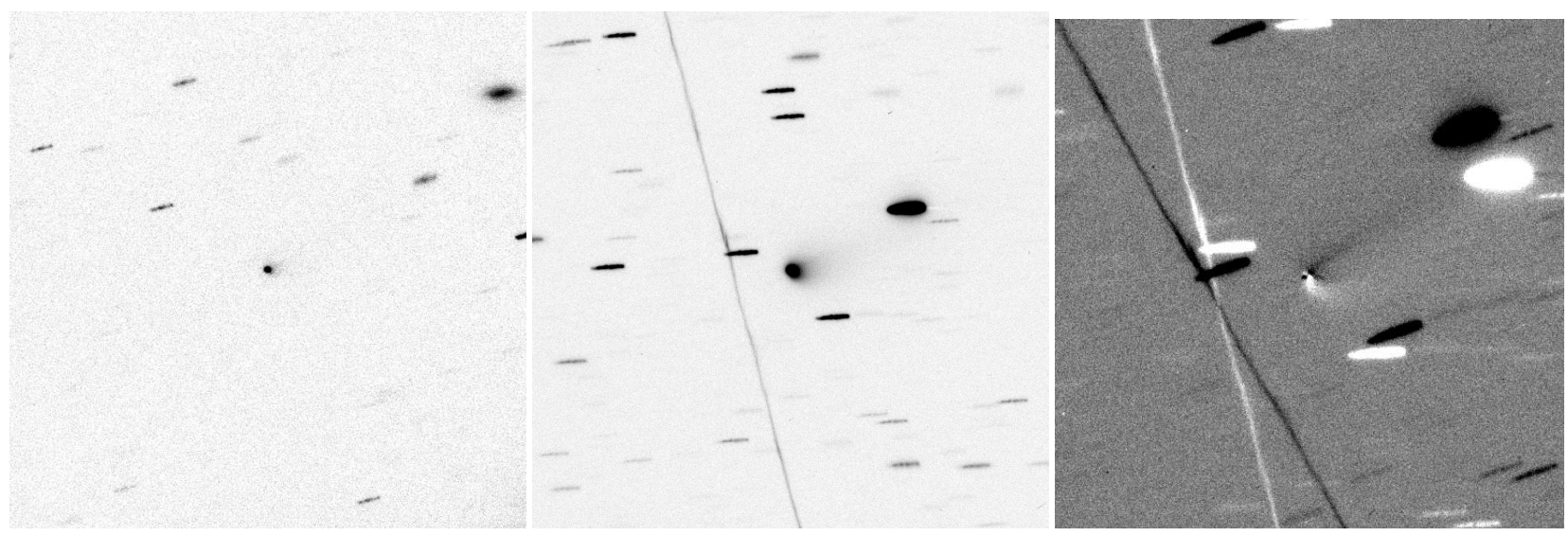

Fig. 2.- Left: 400-sec image of comet 9P/Tempel 1 obtained using the University of Hawaii 2.2-meter telescope on Mauna Kea with a CCD and R filter on December 1, 2004. The comet was at $r=2.45$ AU and $\Delta=2.49 \mathrm{AU}, \alpha=23.0^{\circ}$ at the time. The dust coma extends over $2.0 \times 10^{4} \mathrm{~km}$ to the west. Center: $600-\mathrm{s}$ image of comet 9P/Tempel 1 obtained using the UH 2.2-meter telescope through an R filter on January 18, 2005 when the comet was at $r=2.17 \mathrm{AU}$ and $\Delta=1.65 \mathrm{AU}, \alpha=25.5^{\circ}$. The dust coma extends over $1.1 \times 10^{5} \mathrm{~km}$ to the west. The field of view is $2.5 \times 10^{5} \mathrm{~km}$; north is at top and east is at left for both images. Right: Enhanced image from January 18, 2005, showing the dust jets within the coma. The field of view is $1.5 \times 10^{5} \mathrm{~km}$. 


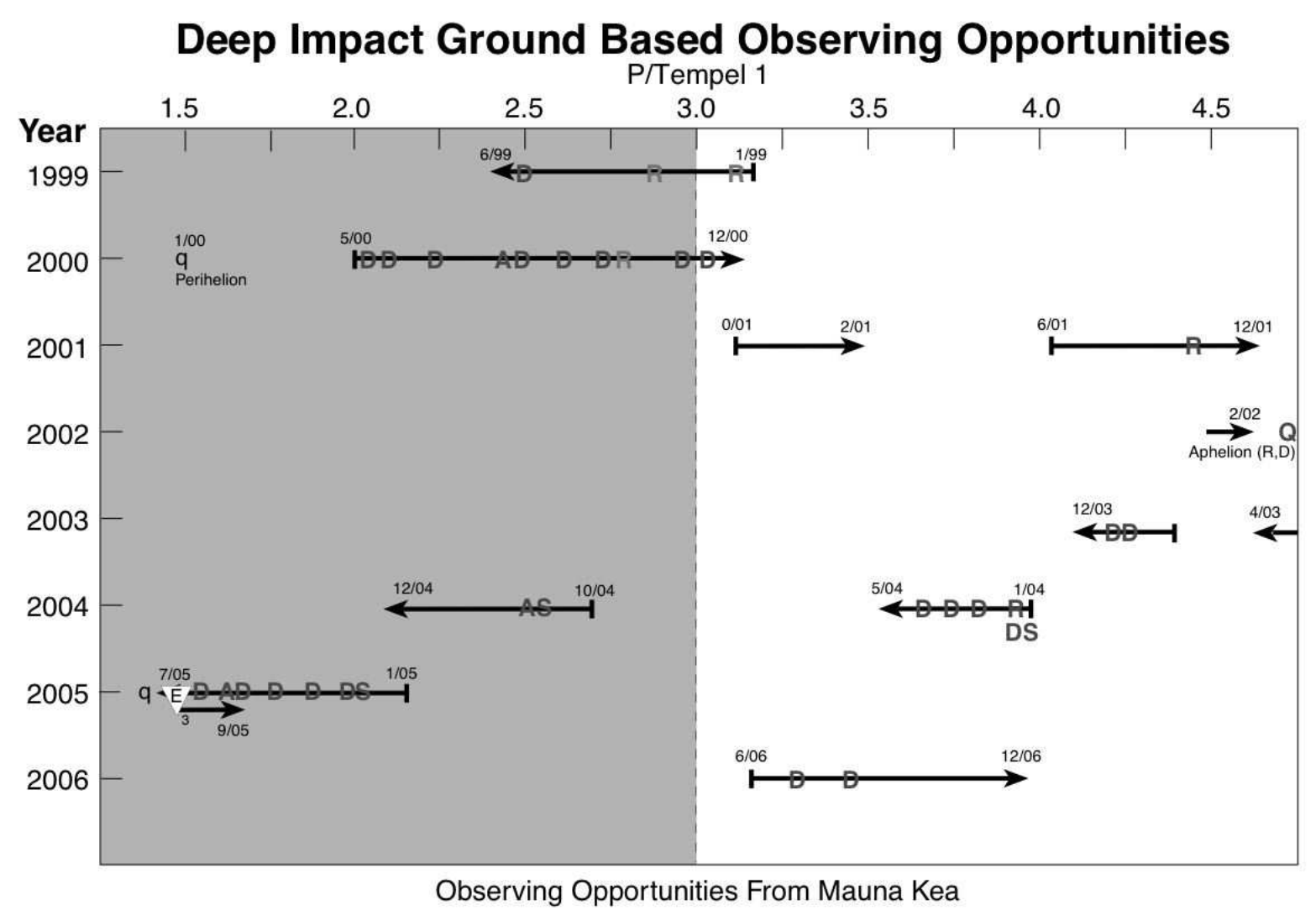

Fig. 3.- Timeline for critical 9P/Tempel 1 observations. Heliocentric distance in AU is shown at top. $\mathbf{R}=$ rotation determination, $\mathbf{A}=$ albedo (simultaneous optical/IR), $\mathbf{S}=$ spectroscopic observations, $\mathbf{D}=$ imaging the dust coma for particle dynamical models. Shading indicates the distances at which significant coma is expected. With the exception of spectroscopy in January 2004, all of the noted observing opportunities have been (or will be) taken advantage of. 


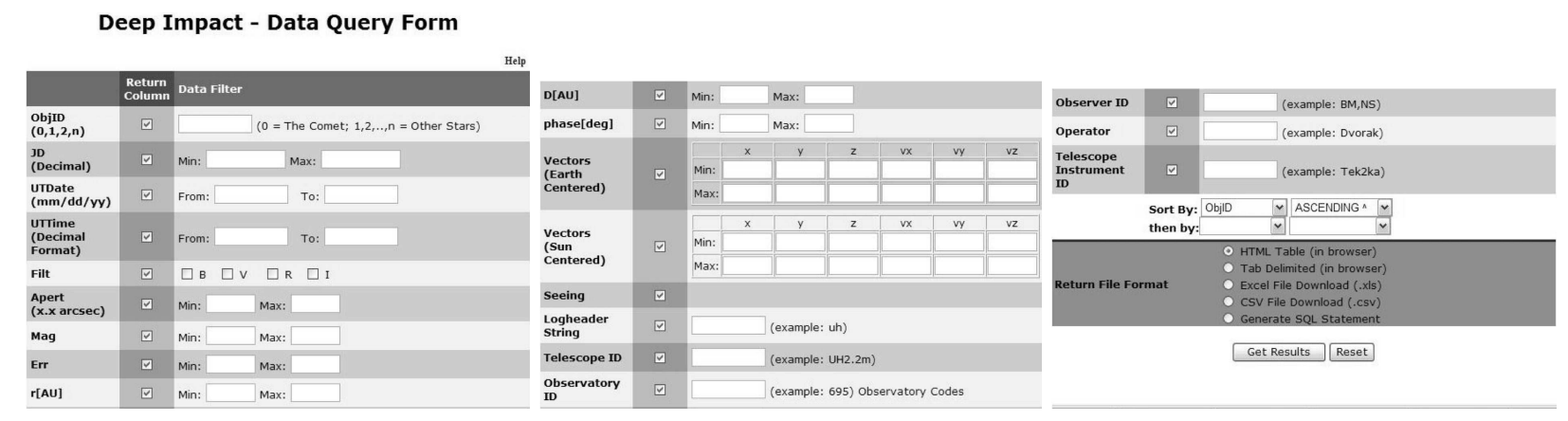

Fig. 4.- Screen shots of the Deep Impact database web browser query form. 


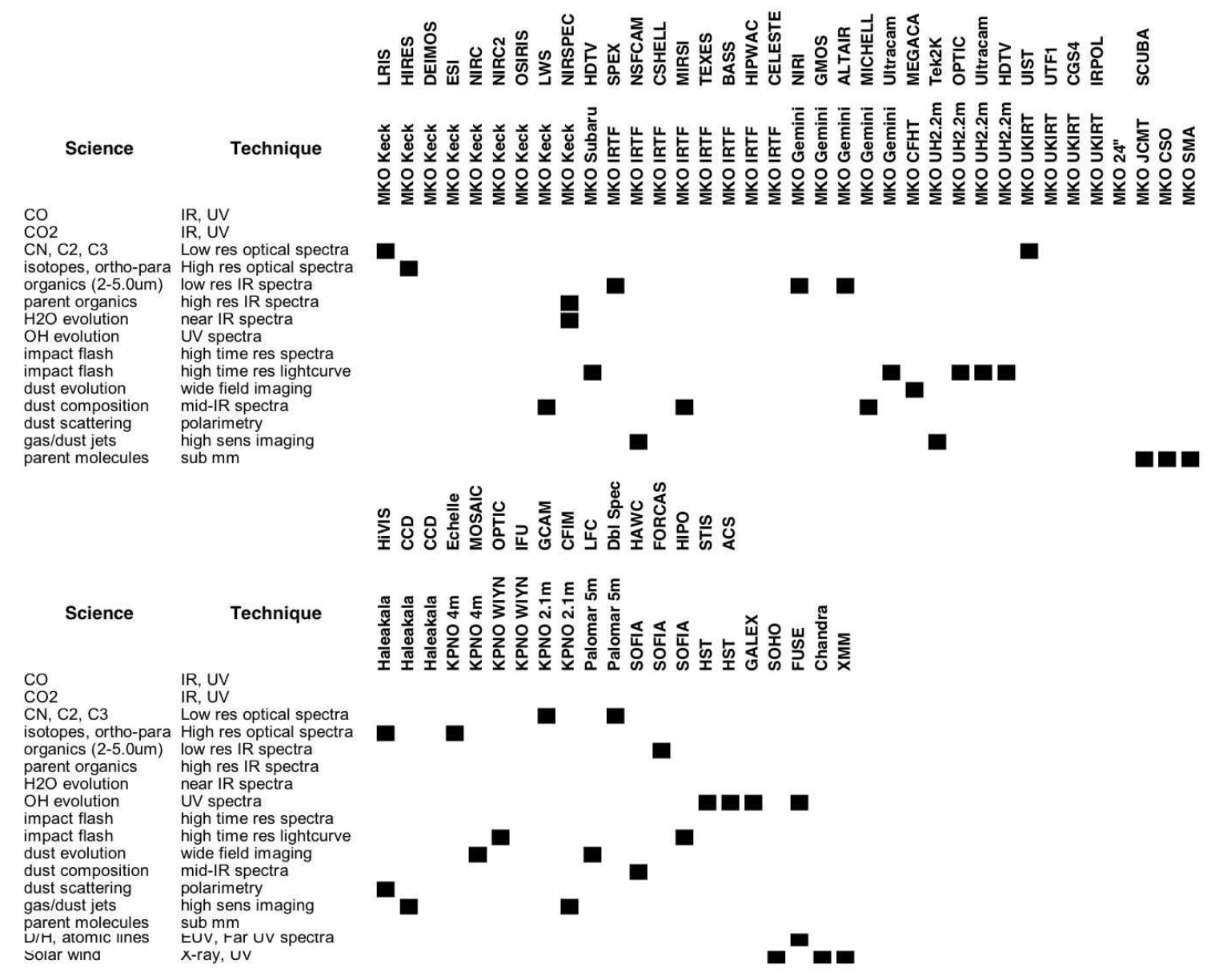

Fig. 5.- Breakdown of wavelengths and science goals that could be potentially covered by specific observatories. 
TABLE 1

Spitzer Space Telescope Observing Opportunities

\begin{tabular}{cccccc}
\hline \hline UT Dates & $r[\mathrm{AU}]^{\dagger}$ & $\Delta[\mathrm{AU}]^{\dagger}$ & $\alpha[\mathrm{deg}]^{\ddagger}$ & Coma? $^{\S}$ & Obs $^{\#}$ \\
\hline $10 / 03 / 03-11 / 13 / 03$ & $4.272-4.157$ & $4.287-3.542$ & $13.4-11.6$ & no & none \\
$02 / 27 / 04-04 / 06 / 04$ & $3.809-3.657$ & $3.281-3.717$ & $13.6-15.6$ & no & awarded \\
$12 / 24 / 04-02 / 23 / 05$ & $2.321-1.967$ & $2.088-1.137$ & $25.6-20.7$ & yes & awarded \\
$07 / 02 / 05-10 / 14 / 05$ & $1.506-1.798$ & $0.882-1.818$ & $41.6-32.0$ & yes & awarded \\
\hline
\end{tabular}

NOTE. - This includes only the windows that satisfy the solar elongation constraints for SIRTF observing. ${ }^{\dagger}$ Heliocentric and geocentric distance; ${ }^{\ddagger}$ Phase angle; ${ }^{\S}$ Existence of observable coma; ${ }^{\#}$ Observing time.

TABLE 2

Optimal Observing Times for Rotation Campaigns

\begin{tabular}{cccccc}
\hline \hline Dates & $r[\mathrm{AU}]^{\dagger}$ & Mag $_{\text {nuc }}$ & ${\text { Elong }[\mathrm{deg}]^{\ddagger}}^{\ddagger}$ & $\alpha[\mathrm{deg}]^{\S}$ & Coma? $^{\mathbb{1}}$ \\
\hline $02 / 99$ & 3.0 & 19.3 & 120 & 9 & probably \\
$10 / 00$ & 2.7 & 18.4 & -9 & 6 & yes \\
$11 / 01$ & 4.3 & 20.8 & 43 & 2 & probably not \\
$12 / 02$ & 4.7 & 21.1 & 70 & 3 & no \\
$12 / 03$ & 4.1 & 22.0 & 100 & 1 & no \\
$04 / 05$ & 1.7 & 15.9 & -169 & 14 & yes \\
\hline
\end{tabular}

Note.-Notes: ${ }^{\dagger}$ Heliocentric distance; ${ }^{\ddagger}$ Solar Elongation; ${ }^{\S}$ Phase angle; "Existence of observable coma; Observations were obtained for all dates prior to 4/05 as of Jan. 2005.

TABLE 3

Phase Function Observing Windows

\begin{tabular}{cccc}
\hline \hline Dates & $r[\mathrm{AU}]$ & $\alpha[\mathrm{deg}]$ & $\operatorname{Mag}_{\text {nuc }}$ \\
\hline $08 / 13 / 01-02 / 02 / 02$ & $4.13-4.53$ & $14.2-1.4$ & $23.5-21.9$ \\
$10 / 01 / 03-01 / 03 / 04$ & $4.27-4.00$ & $13.4-1.8$ & $23.0-21.9$ \\
\hline
\end{tabular}

Note.-Minimum $\alpha$ occurred on $11 / 07 / 01$ at $\alpha=1.27^{\circ}$, and $01 / 02 / 04$ at $\alpha=1.84^{\circ}$. 
TABLE 4

Example Pre-Encounter Emission Line Strengths

\begin{tabular}{ccccccccc}
\hline \hline Species & \multirow{2}{*}{$\mathrm{Ab}^{\dagger}$} & Line & IRAM $\int T d v^{\ddagger}$ & $S / N^{\S}$ & JCMT $\int T d v^{\ddagger}$ & $S / N^{\S}$ & CSO $\int T d v^{\ddagger}$ & $S / N^{\S}$ \\
\hline \multirow{2}{*}{$\mathrm{HCN}$} & $0.1 \%$ & $J=1 \rightarrow 0$ & 27 & 3 & - & - & - & - \\
& & $J=3 \rightarrow 2$ & 210 & 5 & 84 & 6 & 49 & 4 \\
$\mathrm{CO}$ & \multirow{2}{*}{$10 \%$} & $J=4 \rightarrow 3$ & - & - & 86 & 5 & 47 & 2 \\
& & $J=2 \rightarrow 1$ & 19 & 1 & 9 & 1 & 6 & 0.5 \\
$\mathrm{CH}_{3} \mathrm{OH}$ & $3 \%$ & $145 \mathrm{GHz}$ & 31,20 & - & 22 & 1.5 & 15 & 1 \\
& & $157 \mathrm{GHz}$ & $22-10$ & $\sim 1$ & - & - & - & - \\
& & $241 \mathrm{GHz}$ & 71,54 & 3,2 & 41,30 & 3,2 & 30 & - \\
$\mathrm{CS}$ & $04 / 307 \mathrm{GHz}$ & - & - & - & - & 45,54 & 4,5 \\
& $0.1 \%$ & $J=3 \rightarrow 2$ & 21 & 2 & - & - & - & - \\
& & $J=5 \rightarrow 4$ & 51 & 2 & 25 & 2 & 16 & 1 \\
& & $J=7 \rightarrow 6$ & - & - & 22 & 1.5 & 13 & 1 \\
\hline
\end{tabular}

Note.-Predicted line strengths are based on expected gas production rates occuring immediately before impact. ${ }^{\dagger}$ Abundance is expressed as a fraction of the $\mathrm{H}_{2} \mathrm{O}$ abundance. ${ }^{\ddagger}$ Units of line strength are $\mathrm{mK} \mathrm{km} / \mathrm{s}$, with $T=$ temperature in the spectrum across the line as a function of relative speed $v$. ${ }^{\S}$ Signal-to-noise ratio expected for about 2.4 hours of actual integration time (which corresponds to about 4 hours of clock time). 
TABLE 5

Major Participating Observatories - Pre-Encounter Datasets

\begin{tabular}{|c|c|c|c|c|c|}
\hline Year & Telescope & \# Nts & PIs & Observers & Program \\
\hline 1999 & $\mathrm{UH} 2.2 \mathrm{~m}$ & 15 & KM & $\mathrm{KM}, \mathrm{GB}$ & Dust, Rotation \\
\hline \multirow[t]{8}{*}{2000} & $\mathrm{UH} 2.2 \mathrm{~m}$ & 30 & $\mathrm{KM}, \mathrm{JP}, \mathrm{GB}$ & $\mathrm{KM}, \mathrm{JP}, \mathrm{BB}, \mathrm{RW}, \mathrm{CT}, \mathrm{GB}, \mathrm{AE}, \mathrm{YF}, \mathrm{PC}$ & Rotation, Dust, Calib \\
\hline & Keck $10 \mathrm{~m}$ & 3 & MA & $\mathrm{YF}, \mathrm{MB} 2$ & Size, Albedo \\
\hline & Lowell $18 \mathrm{~m}$ & 12 & $\mathrm{MB}$ & $\mathrm{MB}$ & Rotation \\
\hline & McDonald $2.7 \mathrm{~m}$ & 8 & $\mathrm{TF}$ & TF & Rotation \\
\hline & TNG $3.6 \mathrm{~m}$ & 4 & GT & GT & Rotation \\
\hline & Danish $1.5 \mathrm{~m}$ & 5 & TS & TS & Rotation \\
\hline & $\mathrm{ESO} 2.2 \mathrm{~m}$ & 2 & $\mathrm{OH}$ & $\mathrm{OH}, \mathrm{KM}$ & Rotation \\
\hline & VLT $8.0 \mathrm{~m}$ & 1 & $\mathrm{LB}$ & $\mathrm{LB}, \mathrm{KM}, \mathrm{HB}$ & Rotation \\
\hline \multirow[t]{8}{*}{2001} & $\mathrm{UH} 2.2 \mathrm{~m}$ & 42 & $\begin{array}{l}\text { KM, JP, YF, GB, } \\
\text { SS }\end{array}$ & KM, JP, MK, YF, HH, DT, SM, GB, SS & Rotation \\
\hline & CTIO $4 \mathrm{~m}, 1.5 \mathrm{~m}$ & 12 & $\mathrm{RM}, \mathrm{MM}, \mathrm{KK}$ & $\mathrm{RM}, \mathrm{MM}, \mathrm{KK}$ & Rotation \\
\hline & $\mathrm{KPNO} 4 \mathrm{~m}, 2.1 \mathrm{~m}$ & 11 & $\mathrm{PM}, \mathrm{NS}, \mathrm{MB}$ & $\mathrm{PM}, \mathrm{NS}, \mathrm{TL}, \mathrm{MB}$ & Rotation \\
\hline & Lowell $1.8 \mathrm{~m}$ & 3 & $\mathrm{MB}$ & $\mathrm{MB}$ & Rotation \\
\hline & TNG $3.6 \mathrm{~m}$ & 6 & GT & GT, JL & Rotation \\
\hline & McDonald $2.7 \mathrm{~m}$ & 1 & $\mathrm{TF}$ & $\mathrm{TF}$ & Rotation \\
\hline & Bohyunsan x.xm & 1 & YC & YC & Rotation \\
\hline & Wise $1.1 \mathrm{~m}$ & 8 & $\mathrm{YC}$ & $\mathrm{YC}$ & Rotation \\
\hline \multirow[t]{3}{*}{2002} & $\mathrm{UH} 2.2 \mathrm{~m}$ & 20 & $\mathrm{KM}, \mathrm{JP}, \mathrm{YF}$ & $\mathrm{KM}, \mathrm{HH}, \mathrm{JP}, \mathrm{YF}$ & Rotation, Phase Fn \\
\hline & KPNO $2.1 \mathrm{~m}$ & 3 & $\mathrm{BM}, \mathrm{NS}$ & $\mathrm{BM}, \mathrm{NS}$ & Rotation, Phase Fn \\
\hline & TNG $3.6 \mathrm{~m}$ & 4 & GT & $\mathrm{GT}$ & Rotation, Phase Fn \\
\hline \multirow[t]{4}{*}{2003} & $\mathrm{UH} 2.2 \mathrm{~m}$ & 18 & $\begin{array}{l}\mathrm{KM}, \mathrm{JP}, \mathrm{DK}, \mathrm{HH} \text {, } \\
\mathrm{YF}\end{array}$ & $\mathrm{KM}, \mathrm{DH}, \mathrm{JP}, \mathrm{YF}, \mathrm{DK}, \mathrm{HH}$ & PhaseFn \\
\hline & $\mathrm{KPNO} 4 \mathrm{~m}$ & 3 & RM, LW & RM, LW & Phase Fn \\
\hline & CTIO $4 \mathrm{~m}$ & 2 & NS2 & $\mathrm{PC} 2, \mathrm{CA}$ & Phase Fn \\
\hline & NTT $3.6 \mathrm{~m}$ & 1 & $\mathrm{KM}$ & $\mathrm{CF}$ & Phase Fn \\
\hline \multirow[t]{2}{*}{2004} & $\mathrm{UH} 2.2 \mathrm{~m}$ & 10 & $\mathrm{KM}, \mathrm{JP}, \mathrm{YF}$ & JP, KM, YF, NM & Rotation, Phase Fn \\
\hline & VLT 8m & 4 & $\mathrm{KM}$ & KM & Rotation, Phase Fn \\
\hline
\end{tabular}

Note.-Note: Observer affiliations are appropriate to the time of the observations. $\mathrm{AE}=\mathrm{A} . \mathrm{Evans}$; $\mathrm{BB}=\mathrm{B}$. Barris, CT=C. Trujillo, $\mathrm{DC}=\mathrm{D}$. Kocevski, DT=D. Tholen, HH=H. Hsieh, GB=J. Bauer, JP=J. Pittichová, KM=K. Meech, MK=M. Kadooka, NM=N. Moskowitz, $\mathrm{PC}=\mathrm{P}$. Capak, $\mathrm{PH}=\mathrm{P}$. Henry, RW=R. Wainscoat, $\mathrm{YF}=\mathrm{Y}$. Fernández (IfA); $\mathrm{BM}=\mathrm{B}$. Mueller, $\mathrm{CA}=\mathrm{C}$. Aguilera, MB2=M. Belton, NS=N. Samarasinha, NS2=N. Suntzeff, PC2=P. Candia (NOAO); CF=C. Foellmi, HB=H. Boehnhardt, OH=O. Hainaut, TS=T. Sekiguchi $(\mathrm{ESO})$; $\mathrm{LW}=\mathrm{L}$. Wasserman, $\mathrm{MB}=\mathrm{M}$. Buie, $\mathrm{RM}=\mathrm{R}$. Millis (Lowell); DH=D. Hampton (Ball Aerospace); GT=G.-P. Tozzi (Oss. Astrofisico di Arcetri); JL=J. Licandro (Inst. de Astrofisica de Canarias); LB=L. Barrera (Univ. N. Chile); MM=M. Mateo (U. Mich); MA=M. A'Hearn, SM=S. McLaughlin (UMD); TF=T. Farnham (Univ. TX); YC=Y.-J. Choi (Univ. Tel Aviv); 
TABLE 6

WORLD OBSERVABILITY

\begin{tabular}{|c|c|c|c|c|c|c|c|c|}
\hline Observatory & Code $^{£}$ & $\lambda^{\dagger}$ & $\phi^{\ddagger}$ & $\chi^{\S}$ & ATwiBeg & ATwiEnd & $\chi<2.5^{\dagger \dagger}$ & Note \\
\hline Nançay & - & 000:12:00 & $+47: 22: 00$ & Set & $21: 35$ & $22: 50$ & $16: 15-21: 25$ & 1 \\
\hline Meudon & 005 & 002:13:53 & $+48: 48: 18$ & Set & $21: 40$ & $23: 20$ & $16: 20-21: 00$ & 1 \\
\hline Klet & 046 & $014: 17: 17$ & $+48: 51: 48$ & Set & $21: 00$ & $22: 30$ & $15: 30-20: 10$ & 1 \\
\hline Boyden & 074 & 026:24:21 & $-29: 02: 17$ & Set & $16: 30$ & $16: 50$ & $12: 30-21: 30$ & 1 \\
\hline Kiev & 085 & 030:30:08 & $+50: 27: 10$ & Set & $20: 10$ & 00:00 & $14: 40-18: 50$ & 1 \\
\hline Wise & 097 & $034: 45: 45$ & $+30: 35: 44$ & Set & $17: 50$ & $18: 20$ & $12: 50-20: 00$ & 1 \\
\hline Majdanak & 188 & $066: 52: 48$ & $+38: 43: 19$ & Set & $16: 20$ & $16: 50$ & $11: 10-17: 30$ & 1 \\
\hline Kavalur & 220 & 078:49:35 & $+12: 34: 34$ & Set & $14: 10$ & $14: 30$ & $09: 30-17: 40$ & 1 \\
\hline Yunnan & 286 & $102: 47: 24$ & $+25: 01: 32$ & Set & $13: 10$ & $13: 30$ & $08: 10-15: 40$ & 1 \\
\hline Perth & 323 & 116:08:06 & $-32: 00: 31$ & 3.0 & $10: 30$ & $10: 50$ & $06: 40-13: 50$ & 2 \\
\hline Purple Mtn & 330 & $118: 49: 15$ & $+32: 04: 00$ & 5.2 & $12: 20$ & $12: 50$ & $07: 20-14: 20$ & 2 \\
\hline Lulin & D35 & $120: 52: 25$ & $+23: 28: 07$ & 3.8 & $11: 50$ & $12: 10$ & $06: 50-14: 40$ & 2 \\
\hline Bohyunsan & 344 & $128: 58: 36$ & $+36: 09: 53$ & 3.4 & $12: 00$ & $12: 30$ & $06: 50-13: 40$ & 2 \\
\hline Siding Spring & 260 & $149: 03: 58$ & $-31: 16: 37$ & 1.4 & $08: 10$ & $08: 30$ & $04: 20-13: 20$ & 2 \\
\hline Mt. John & 474 & $170: 27: 54$ & $-43: 59: 15$ & 1.3 & $06: 30$ & $06: 50$ & $03: 10-11: 50$ & 3 \\
\hline Mauna Kea & 568 & 204:31:40 & $+19: 49: 34$ & 1.2 & 06:00 & $06: 20$ & 01:10-09:10 & 4 \\
\hline Haleakala & 608 & 203:44:31 & $+20: 42: 30$ & 1.2 & 06:00 & $06: 20$ & $01: 10-09: 10$ & 4 \\
\hline San Pedro & 679 & $224: 32: 11$ & $+31: 02: 39$ & 2.5 & $03: 55$ & $04: 25$ & $22: 50-06: 15$ & 5 \\
\hline Palomar & 675 & $243: 08: 28$ & $+33: 21: 26$ & 2.5 & 04:10 & 04:40 & $23: 10-06: 10$ & 5 \\
\hline Goldstone & - & $243: 12: 18$ & $+35: 14: 48$ & 2.6 & $04: 20$ & $04: 50$ & $23: 10-06: 10$ & 6 \\
\hline KPNO & 695 & $248: 24: 19$ & $+31: 57: 32$ & 2.9 & $03: 40$ & 04:10 & $22: 40-05: 50$ & 6 \\
\hline Lowell & 688 & $248: 27: 52$ & $+35: 05: 46$ & 3.1 & 04:00 & $04: 30$ & $22: 50-05: 40$ & 6 \\
\hline McDonald & 711 & $255: 58: 43$ & $+30: 40: 17$ & 3.8 & 03:10 & $03: 30$ & $22: 10-05: 20$ & 6 \\
\hline CTIO & 807 & $289: 11: 39$ & $-30: 10: 09$ & Set & $23: 00$ & $23: 20$ & 19:00-04:00 & 1 \\
\hline Pachon & - & $289: 15: 48$ & $-30: 14: 26$ & Set & $23: 00$ & $23: 20$ & 19:00-04:00 & 1 \\
\hline $\mathrm{ESO}$ & 809 & $289: 16: 13$ & $-29: 15: 26$ & Set & $23: 00$ & $23: 20$ & 19:00-04:00 & 1 \\
\hline Paranal & 309 & $289: 35: 48$ & $-24: 37: 32$ & Set & $23: 00$ & $23: 20$ & 19:00-04:00 & 1 \\
\hline Arecibo & 251 & 293:14:49 & $+18: 20: 36$ & Set & $14: 00$ & $14: 20$ & $09: 50-18: 50$ & 1 \\
\hline La Palma & 950 & $342: 07: 03$ & $+28: 45: 37$ & Set & $21: 20$ & $21: 40$ & $16: 20-23: 40$ & 1 \\
\hline Armagh & 981 & $353: 21: 08$ & $+54: 21: 10$ & Set & $23: 40$ & 01:40 & $17: 50-20: 30$ & 1 \\
\hline IRAM-30m & J86 & $356: 36: 26$ & $+37: 03: 58$ & Set & $20: 45$ & $21: 30$ & $15: 40-22: 25$ & 1 \\
\hline
\end{tabular}

Note. $-{ }^{£}$ Minor Planet Center observatory code; ${ }^{\dagger}$ East longitude; ${ }^{\ddagger}$ Geocentric latitude; ${ }^{\S}$ Airmass at time of encounter, nominally 06:10 UT on 4 July 2005; " UT at beginning and end of astronomical twilight closest to observability window; ${ }^{\dagger \dagger} \mathrm{UT}$ time interval that the comet is observable at airmasses $\chi<2.5$. Notes: $1-$ Not above horizon at impact time; 2-Above horizon at impact time, but in daytime; 3-Above horizon at impact time, but in civil or nautical twilight; 4-Above horizon at impact time, but in astronomical twilight; 5-Above horizon at impact time, and in darkness, and at $\chi<2.5 ; 6$-Above horizon at impact time, and in darkness, but at $\chi \geq 2.5$. 
TABLE 7

Observing Workshops and Meetings for 9P/Tempel 1 Coordination

\begin{tabular}{lccl}
\hline \hline \multicolumn{1}{c}{ Workshop } & Dates & \#People & \multicolumn{1}{c}{ Location } \\
\hline Division for Planetary Sciences & $09 / 01 / 03$ & 50 & Monterey, CA \\
European Southern Observatory & $02 / 14-15 / 04$ & 17 & ESO HQ, Garching \\
NOAO/KPNO & $02 / 20 / 04$ & 20 & NOAO HQ, Tucson, AZ \\
Institute for Astronomy & $04 / 30 / 05$ & 38 & IfA Hilo, Hawaii \\
National Central University & $05 / 28 / 04$ & 30 & NCU, Jhong-Li City, Taiwan \\
American Astron. Society Mtg. & $05 / 30 / 04$ & 4 & Denver, CO \\
NOAO/CTIO & $06 / 24 / 04$ & 110 & NOAO HQ, La Serena, Chile \\
Anglo Australian Observatory & $09 / 06 / 04$ & 22 & Macquarie Univ., Sydney Australia \\
\hline
\end{tabular}

TABLE 8

Earth-Based Encounter Science

\begin{tabular}{|c|c|c|c|}
\hline Science Goal & Wavelength & Details & Comments \\
\hline Parent Volatiles & $\begin{array}{c}\text { UV } \\
\text { IR } 2-5 \mu m \\
\text { IR } 5-9 \mu m \\
\text { Sub-mm, mm }\end{array}$ & $\begin{array}{l}\mathrm{CO}, \mathrm{CO}_{2}, \mathrm{H}_{2} \mathrm{O} \\
\mathrm{H}_{2} \mathrm{O}, \text { many organics } \\
\text { organics, PAH } \\
\mathrm{H}_{2} \mathrm{O}, \mathrm{HCN}, \mathrm{OH}, \mathrm{CS}\end{array}$ & $\begin{array}{l}\mathrm{CO}_{2} \text { requires modeling } \mathrm{CO} \text { Cameron band obser- } \\
\text { vations } \\
\text { High resolution needed for many molecules } \\
\text { Cannot be done from ground; new regime }\end{array}$ \\
\hline Isotopic Abundances & $\begin{array}{l}\text { Optical } 0.3-0.9 \mu m \\
\text { UV \& Radio }\end{array}$ & $\begin{array}{l}{ }^{14} \mathrm{~N} /{ }^{15} \mathrm{~N},{ }^{13} \mathrm{C} /{ }^{14} \mathrm{C} \\
\mathrm{D} / \mathrm{H}\end{array}$ & Requires high resolution spectroscopy \\
\hline Ortho-Para Ratios & $\begin{array}{c}\text { Optical } 0.3-0.9 \mu m \\
\text { IR }\end{array}$ & $\begin{array}{l}\text { for } \mathrm{NH}_{2} \\
\mathrm{H}_{2} \mathrm{O}\end{array}$ & Requires high resolution $\rightarrow$ spin temperature \\
\hline Dust Evolution & $\begin{array}{l}\text { Optical } 0.3-0.9 \mu m \\
\text { IR } 9-45 \mu m\end{array}$ & $\begin{array}{l}\text { Particle sizes } \\
\text { silicate evolution }\end{array}$ & Wide field Imaging \& dust dynamical models \\
\hline
\end{tabular}


TABle 9

Observing Allocations as of January 2005

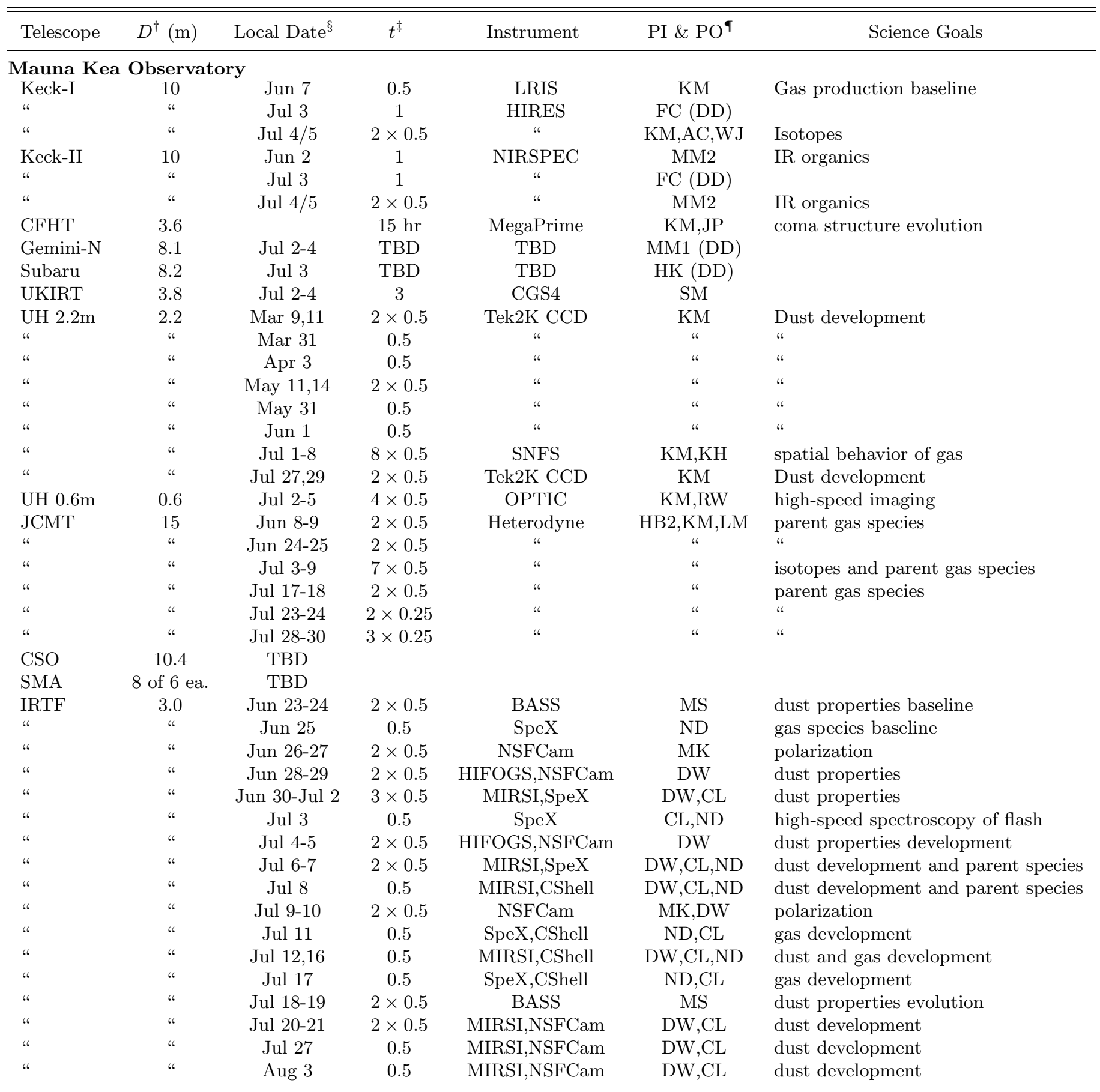


TABLE 9-Continued

\begin{tabular}{|c|c|c|c|c|c|c|}
\hline Telescope & $D^{\dagger}(\mathrm{m})$ & Local Date $^{\S}$ & $t^{\ddagger}$ & Instrument & $\mathrm{PI} \& \mathrm{PO}^{\llbracket}$ & Science Goals \\
\hline \multicolumn{7}{|c|}{ Space-based Observatories } \\
\hline Spitzer & 0.85 & Feb 3 & $1.7 \mathrm{hr}$ & MIPS & CL & large dust grain environment \\
\hline$"$ & " & Feb 6 & $5.3 \mathrm{hr}$ & IRS & CL & " \\
\hline " & " & Feb 10 & $0.4 \mathrm{hr}$ & IRS & CL & high-speed spectroscopy test \\
\hline " & “ & Jul 2-4 & $10 \mathrm{hr}$ & IRS & CL & high-speed spectroscopy, dust imaging \\
\hline Hubble & 2.4 & Jul 3-4 & & ACS & $\mathrm{PF}$ & high-resolution imaging \\
\hline Chandra & 0.4 & Jul 2-5 & $200 \mathrm{ks}$ & ACIS-S & CL & spectroscopy \\
\hline XMM & 0.4 & Jul 3-4 & $63 \mathrm{ks}$ & EPIC,RGS,OM & $\mathrm{RS}$ & simultaneous $x$-ray and optical data \\
\hline GALEX & 0.5 & Jul 3-5 & $150 \mathrm{ks}$ & spectrometer & $\mathrm{PF}$ & $\mathrm{CO}, \mathrm{CO}_{2}$ \\
\hline \multicolumn{7}{|c|}{ National Optical Astronomy Observatories } \\
\hline Blanco & 4 & Jul $3-6$ & $4 \times 0.5$ & ISPI & $\mathrm{KM}, \mathrm{DH}$ & dust color and evolution \\
\hline CTIO $1.5 \mathrm{~m}$ & 1.5 & Jul 3-6 & $4 \times 0.5$ & CPAPIR & KM & dust color simultaneity \\
\hline CTIO $0.9 \mathrm{~m}$ & 0.9 & Jul 3-6 & $4 \times 0.5$ & CFIM CCD & KM & dust color simultaneity \\
\hline CTIO Schmidt & & Jul 3-5 & $3 \times 0.5$ & & PS & wide field dust \\
\hline Mayall & 4 & Jul 1-8 & 8 & MOSAIC & MA & narrowband imaging \\
\hline KPNO $2.1 \mathrm{~m}$ & 2.1 & Feb 1-4 & 4 & CFIM CCD & MA & dust development baseline \\
\hline$"$ & " & Mar 10-13 & 4 & " & " & " \\
\hline " & " & Apr 8-13 & 4 & " & “ & “ \\
\hline " & " & Jun $3-6$ & 4 & " & " & $"$ \\
\hline " & " & Jul 1-8 & 8 & SQIID & " & near-infrared dust imaging \\
\hline WIYN & 3.5 & Jul 2-6 & 5 & DSPKB & $\mathrm{BM}$ & integral-field spectra \\
\hline SOAR & 4.1 & & & Phoenix & & \\
\hline Gemini-S & 8.1 & Jul 2-4 & TBD & TBD & MM1 (DD) & \\
\hline " & " & queue & $5 \mathrm{hr}$ & T-ReCs & JD & dust silicate properties \\
\hline \multicolumn{7}{|c|}{ European Southern Observatory } \\
\hline VLT Antu & 8.2 & Jul $2-10$ & $8 \times 0.5$ & FORS2 & HR & gas coma daughter species \\
\hline VLT Kueyen & 8.2 & Jul $10-12$ & $2 \times 0.5$ & FORS1 & HB1 & polarization \\
\hline$"$ & $"$ & queue & $10 \mathrm{hr}$ & UVES & $\mathrm{HR}$ & high-resolution spectroscopy, isotopes \\
\hline " & " & Jul $2-10$ & $8 \times 0.5$ & " & " & " \\
\hline VLT Melipal & 8.2 & Jul 2-6 & $4 \times 0.5$ & VISIR & HB1 & dust silicate evolution \\
\hline VLT Yepun & 8.2 & Jul 2-10 & $8 \times 0.5$ & NACO & " & near-IR coma structure \\
\hline ESO $3.6 \mathrm{~m}$ & 3.6 & Jul 2-10 & $8 \times 0.5$ & TIMMI2 & " & dust silicate evolution \\
\hline NTT & 3.6 & Apr-Jun & $3 \times 0.5$ & EMMI & $\mathrm{OH}$ & imaging (dust scattering), spectra (gas) \\
\hline$"$ & " & May 31-Jun 2 & $3 \times 0.4$ & EMMI & " & imaging (dust scattering), spectra (gas) \\
\hline " & " & Jul $2-7$ & $5 \times 0.5$ & SOFI & " & near-IR coma structure \\
\hline$"$ & $"$ & Jul $7-10$ & $3 \times 0.5$ & EMMI & " & imaging for dust scattering properties \\
\hline $\mathrm{ESO} 2.2 \mathrm{~m}$ & 2.2 & Jul 2-10 & $8 \times 0.5$ & WFI & " & imaging for dust scattering properties \\
\hline \multicolumn{7}{|c|}{ Observatorio del Roque de los Muchachos - La Palma } \\
\hline WHT & 4.2 & Jul 3-7 & $5 \times 0.5$ & OASIS/LCRIS & $\mathrm{JL}$ & opt spectra, near IR imaging \\
\hline TNG & 3.6 & Jul 1-8 & $8 \times 0.5$ & DOLORES/SARG & $\mathrm{JL}$ & opt/near-IR spectra \\
\hline NOT & 2.5 & Jul 1-8 & $8 \times 0.5$ & ALFOSC & JL & low res spectra - gas \\
\hline INT & 2.5 & Jul 1-7 & $7 \times 0.5$ & WFC & $\mathrm{C}$ & imaging for dust scattering properties \\
\hline \multicolumn{7}{|c|}{ Calar Alto Observatory } \\
\hline & 2.2 & Apr & 5 & CAFOS & $\mathrm{LL}$ & optical spectroscopy \\
\hline & 2.2 & Jan-Jul 1 & & CAFOS & LL & RI images every $2-3$ days \\
\hline
\end{tabular}


TABLE 9-Continued

\begin{tabular}{|c|c|c|c|c|c|c|}
\hline Telescope & $D^{\dagger}(\mathrm{m})$ & Local Date ${ }^{\S}$ & $t^{\ddagger}$ & Instrument & $\mathrm{PI} \& \mathrm{PO}^{\top}$ & Science Goals \\
\hline & 2.2 & Jan-Jul 1 & & CAFOS & LL & monthly optical spectroscopy \\
\hline \multicolumn{7}{|c|}{ Las Campanas Observatory } \\
\hline Baade & 6.5 & Jun 20-21 & $2 \times 0.5$ & PANIC & $\mathrm{DO}$ & near-IR imaging for dust \\
\hline Baade & 6.5 & TBD & TBD & PANIC & $\mathrm{DO}$ & near-IR imaging for dust \\
\hline Clay & 6.5 & TBD & TBD & MAGIC & DO & optical imaging for dust \\
\hline duPont & 2.5 & Jun 28-Jul 10 & $13 \times 0.5$ & WFCCD & $\mathrm{DO}, \mathrm{KVB}$ & optical imaging for dust \\
\hline Swope & 1.0 & Jul 3-8 & $6 \times 0.5$ & Retrocam & KVB & spectroscopy \\
\hline \multicolumn{7}{|c|}{ Lowell Observatory } \\
\hline Perkins & 1.8 & TBD & & MIMIR & $\mathrm{MB}$ & high-speed spectroscopy \\
\hline Hall & 1.1 & TBD & & SITe CCD & & narrowband imaging \\
\hline 31-Inch & 0.8 & $\mathrm{TBD}$ & & Loral CCD & & dust development \\
\hline \multicolumn{7}{|c|}{ Palomar Observatory } \\
\hline Hale & 5 & Jul $2-6$ & $5 \times 0.5$ & PHARO & $\mathrm{JB} 2, \mathrm{RD}$ & high resolution imaging \\
\hline \multicolumn{7}{|c|}{ Anglo-Australian Observatory } \\
\hline AAT & 3.9 & Jul $1-8$ & $8 \times 0.5$ & UCLES & JB1 & high-res. visible spectroscopy \\
\hline UKST & 1.2 & Jul 1-8 & $8 \times 0.5$ & $6 \mathrm{dF}$ & $\mathrm{C}$ & multi-object spectroscopy \\
\hline \multicolumn{7}{|c|}{ Lick Observatory } \\
\hline Shane & 3.0 & Jul $1-5$ & 5 & & $\mathrm{RP}$ & spectroscopy of flash and coma \\
\hline Nickel & 1.0 & TBD & & & & \\
\hline \multicolumn{7}{|c|}{ McDonald Observatory } \\
\hline HET & 9.2 & TBD & & & & \\
\hline Smith & 2.7 & TBD & & & & \\
\hline \multicolumn{7}{|c|}{ Table Mountain Facility } \\
\hline 24-inch & 0.4 & TBD & & & & \\
\hline \multicolumn{7}{|c|}{ Mount Laguna Observatory } \\
\hline 40-inch & 1.0 & TBD & & & & \\
\hline \multicolumn{7}{|c|}{ Observatorio Astronómico Nacional de San Pedro Martir } \\
\hline & 1.5 & TBD & & & & \\
\hline
\end{tabular}

Note. $-^{\dagger} D=$ diameter of primary mirror in meters. $\S$ Observing run starts on this date in 2005, local time. $\ddagger t=$ time allocated, in units of nights unless otherwise stated; $n \times m$ indicates only a part of each night $(m)$ has been allocated over $n$ nights. " PI = Principal Investigator; PO = Primary Observer; "DD" indicated Director's Discretionary time has been assigned. Initials correspond to: MA = Michael A'Hearn, JB1 = Jeremy Bailey, JB2 = James Bauer, MB = Marc Buie, HB1 = Hermann Boehnhardt, HB2 = Harold Butner, FC = Fred Chaffee, C $=$ Coates, $\mathrm{AC}=$ Anita Cochran, JD = James De Buizer, RD = R. Dekany, ND = Neil Dello Russo, PF $=$ Paul Feldman, $\mathrm{OH}=$ Olivier Hainaut, $\mathrm{DH}=$ Doug Hamilton, $\mathrm{KH}=$ Klaus Hodapp, WJ $=$ William Jackson, HK $=$ Hiroshi Karoji, MK = Michael Kelley, LL = Luisa-Maria Lara, JL = Javier Licandro, CL = Carey Lisse, KM = Karen Meech, SM = Steven Miller MM1 = Matt Mountain, BM = Beatrice Mueller, MM2= Michael Mumma, DO $=$ Dave Osip JP = Jana Pittichova, RP = Richard Puetter, HR = Heike Rauer, RS = Rita Schulz, MS = Michael Sitko, KV = Kaspar von Braun, RW = Richard Wainscoat, DW = Diane Wooden, LW = Laura Woodney. 